\title{
Positive eigenvectors and simple nonlinear maps
}

\author{
Marta Calanchi and Carlos Tomei
}

\begin{abstract}
For linear operators $L, T$ and nonlinear maps $P$, we describe classes of simple maps $F=I-P T, F=L-P$ between Banach and Hilbert spaces, for which no point has more than two preimages. The classes encompass known examples (homeomorphisms, global folds) and the weaker, geometric, hypotheses suggest new ones. The operator $L$ may be the Laplacian with various boundary conditions, as in the original Ambrosetti-Prodi theorem, or the operators associated with the quantum harmonic oscillator, the hydrogen atom, a spectral fractional Laplacian, elliptic operators in non-divergent form. The maps $P$ include the Nemitskii map $P(u)=f(u)$ but may be non-local, even non-variational. For self-adjoint operators $L$, we employ familiar results on the nondegeneracy of the ground state. On Banach spaces, we use a variation of the Krein-Rutman theorem.
\end{abstract}

Keywords: Ambrosetti-Prodi theorem, folds, Krein-Rutman theorem, positivity preserving semigroups.

MSC-class: 35J65, 47H11, 47H30, 58K05.

\section{Introduction and main results}

The celebrated Ambrosetti-Prodi theorem provides a geometric description of a class of differential maps. We state it in the context of Sobolev spaces ([1], [2], [8]). For a smooth, bounded domain $\Omega \subset \mathbb{R}^{n}$, denote by $\lambda_{1}<\lambda_{2}$ the two smallest eigenvalues of the (Dirichlet) Laplacian $-\Delta_{D}: D=H^{2}(\Omega) \cap H_{0}^{1}(\Omega) \rightarrow L^{2}(\Omega)$ and by $\phi_{1}>0$ an eigenvector associated with $\lambda_{1}$.

Theorem 1 (Ambrosetti-Prodi). Let $\mathbf{F}: D \rightarrow L^{2}(\Omega), \mathbf{F}(u)=-\Delta_{D} u-\mathbf{f}(u)$, where $\mathbf{f}: \mathbb{R} \rightarrow \mathbb{R}$ is a $C^{2}$ strictly convex function for which

$$
\mathbf{a}=\inf \mathbf{f}^{\prime}(x)<\lambda_{1}<\lambda_{1}+\mathbf{b}=\sup \mathbf{f}^{\prime}(x)<\lambda_{2} .
$$

Then $\mathbf{F}$ folds downwards with respect to $\phi_{1}$. 
We define folds. Given real Banach spaces $X$ and $Y$ and $\phi \in X \cap Y, \phi \neq 0$, let $V=\langle\phi\rangle$ be the subspace generated by $\phi$. Denote the vertical line through $y \in Y$ by $L_{y}=\{y+h \phi, h \in \mathbb{R}\} \subset Y$. A continuous function $F: X \rightarrow Y$ is simple if there is a subspace decomposition $X=W_{X} \oplus V$ with the following property: for each $y \in Y$, the inverse $F^{-1}\left(L_{y}\right)$ is a curve ( a fiber)

$$
\Lambda_{y}=\left\{u(y, t)=w(y, t)+t \phi, w(y, t) \in W_{X}, t \in \mathbb{R}\right\}
$$

such that, for $y+h(y, t) \phi=F(u(y, t))$, the height $h(y,):. \mathbb{R} \rightarrow \mathbb{R}$ is either strictly monotonical or strictly unimodal for each $y \in Y$. Thus, no point has three preimages under $F$. The definition clearly does not depend on the choice of $W_{X}$.

The function $F$ folds downwards with respect to $\phi$ if additionally, for all $y \in Y, h(y, t) \rightarrow-\infty$ as $t \rightarrow \pm \infty$. Then, there is a height $h(y)$ such that $F(u)=y+h \phi$ has zero, one or two preimages, depending if $h>h(y), h=h(y)$ or $h<h(y)$. Similarly, $F$ folds upwards if the inequalities are reversed and $F$ folds folds vertically if either case happens.

Manes and Micheletti stated the theorem with the hypotheses above in the context of Hölder spaces ([25]). With different techniques, Berger and Podolak provided a different proof ([8], also [7]) for Hilbert spaces. The theorem was a starting point of an active line of research, leading to characterizations of folds and further examples. The interested reader may find a rich collection of examples and techniques in the meticulous review papers by Church and Timourian ([15], [16]), and Ruf ([30]), covering the material up to the mid nineties. Their approach is strongly influenced by the original Ambrosetti-Prodi view of the problem: a combination of local theory (the fold being the first, simplest singularity) with global information related to the determination of the number of solutions in terms of some parameter or forcing terms. Such results are frequently restricted by technical requirements, such as smoothness: in this paper, we present operational alternatives and provide generalizations to a wider class of problems.

Recently, Sirakov et alii. ([36]) considered folds given by nonlinear perturbations of second order operators $\mathbf{L}$ in non-divergence form. For a bounded $C^{1,1}$ domain $\Omega \subset \mathbb{R}^{n}, n \geq 2, X=W^{2, n}(\Omega) \cap W_{0}^{1, n}(\Omega), Y=W^{0, n}(\Omega)=L^{n}(\Omega)$, set

$$
\mathbf{L} u=-A_{i j} \partial_{i} \partial_{j} u-b B_{i} \partial_{i} u-q u=-\operatorname{tr}\left(A D^{2} u\right)-B \nabla u-q u,
$$

where, for appropriate constants $\Lambda \geq \lambda>0$, the matrix $A=\left(A_{i j}\right)$ satisfies $A(x) \in C(\bar{\Omega}), \sigma(A(x)) \subset[\lambda, \Lambda]$ and $|B|_{\infty},|q|_{\infty} \leq C$. Then $\mathbf{L} \in \mathcal{B}(X, Y)$, the space of bounded linear operators from $X$ to $Y$. Let $\lambda_{1}=\lambda_{1}(\mathbf{L})$ be the eigenvalue of $\mathbf{L}$ of smallest real part and $\phi_{1}>0$ an associated eigenvector.

Theorem 2 (Sirakov-Tomei-Zaccur). Let $\mathbf{L}$ as above and $\mathbf{P}(u)=\mathbf{f}(u)$ for a strictly convex function $\mathbf{f}: \mathbb{R} \rightarrow \mathbb{R}$ such that

$$
\mathbf{a}=\inf _{r \neq s} \frac{f(r)-f(s)}{r-s}<\lambda_{1}<\lambda_{1}+\mathbf{b}=\sup _{r \neq s} \frac{f(r)-f(s)}{r-s} .
$$


Then, for some $\mathbf{b}>0, \mathbf{F}=\mathbf{L}-\mathbf{P}: X \rightarrow Y$ folds downwards with respect to $\phi_{1}$.

The proof in [36] combines elliptic estimates and geometric arguments. We phrase an extension, Theorem 5, as a geometric statement with a geometric proof.

Both results are inspirational, in the specification of hypotheses and in the techniques of proof. Our results are more geometric and more general. We take a different point of view: simple maps are obtained from nonlinear perturbations of special linear operators. As for the theorems above, the positivity of an eigenvector of the Jacobian, or some appropriate linearization, is an essential ingredient. We use the preservation of positive eigenfunctions associated with the spectral radius (Krein-Rutman type arguments, as in Section 2), or with the smallest eigenvalue of a self-adjoint map (the perturbation theory of nondegenerate groundstates, Section 3). On a global scale, the asymptotic linearity of the nonlinear terms yields a certain uniformity of the linearizations, which belong to a set with privileged compactness properties.

Let $Y$ be a real, separable, reflexive Banach space endowed with a normal, generating cone $K \subset Y$ (Section 2.1 presents the terminology associated with cones). Denote by $k \geq 0$ an element $k \in K$ and by $k>0$ a quasi-interior point $k \in K$. For a bounded operator $S: Y \rightarrow Y$, (i.e. $S \in \mathcal{B}(Y)$ ), denote its spectrum and spectral radius by $\sigma(S)$ and $r(S)$. An operator $S \in \mathcal{B}(Y)$ is r-special if it compact and ergodic with respect to $K$ and $r(S)>0$.

Consider a r-special operator $T \in \mathcal{B}(Y)$ with $r(T)=1$. For $b, R>0$ and an r-special operator $S \in \mathcal{B}(Y)$, define the set of perturbations

$$
\begin{gathered}
\mathscr{P}^{b}=\mathscr{P}_{T}^{R, b, S}=\{H=A+B, \text { for } A, B \in \mathcal{B}(Y),\|A\| \leq R,\|B\| \leq b, \\
S \leq A T \leq T, B \geq 0\}
\end{gathered}
$$

The map $P: Y \rightarrow Y$ admits linearizations if for $y, z \in Y$, there exists $G(y, z)=G(z, y) \in \mathcal{B}(Y)$ for which $P(y)-P(z)=G(y, z)(y-z)$.

The proof of Theorem 1 in [2] uses linearizations. Linearizations $G(y, z) \in$ $\mathcal{B}(Y)$ are not necessarily continuous in $y, z \in Y$.

We enumerate possible properties of $P: Y \rightarrow Y$. Let $F: Y \rightarrow Y, F=I-P T$.

$(\mathrm{r}-\mathrm{H}) \quad P: Y \rightarrow Y$ is a Lipschitz map admitting linearizations $G(y, z) \in \mathscr{P}^{b}$.

(r-Conv) Let $y_{1}, y_{2}, z_{1}, z_{2} \in Y$ such that $y_{1}>z_{1}, y_{2}>z_{2}, y_{1} \geq y_{2}, z_{1} \geq z_{2}$ and either $y_{1}>y_{2}$ or $z_{1}>z_{2}$. Then $G\left(y_{1}, z_{1}\right)-G\left(y_{2}, z_{2}\right) \in \mathcal{B}(Y)$ is strictly positive.

(r-Hs) $P: Y \rightarrow Y$ is a $C^{1}$ map whose Jacobians $J(y)=D P(y)$ belong to $\mathscr{P}^{b}$.

(r-Convs) Let $y, z \in Y, y>z$. Then $D P(y)-D P(z) \in \mathcal{B}(Y)$ is strictly positive.

(r-Crit) $P: Y \rightarrow Y$ is a $C^{1}$ map and some $y \in Y$ is critical: $0 \in \sigma(D F(y))$. 
Theorem 3. Suppose $Y$ and $K$ as above, $T \in \mathcal{B}(Y)$ an r-special operator for which $r(T)=1, T \phi=\phi>0$ and $P: Y \rightarrow Y$ a Lipschitz map. Define the function $F: Y \rightarrow Y, y \mapsto y-P(T y)$. For small $b>0$,

(i) suppose that $(r-H)$ holds and, for $y, z \in Y, r(G(T y, T z) T) \neq 1$. Then $F$ is a homeomorphism onto its image;

(ii) suppose that $(r-H),(r-C o n v)$ hold. Then $F$ is a simple map;

(iii) if $(r-H),(r$-Conv) hold and $F: Y \rightarrow Y$ is proper, then it is a homeomorphism or folds vertically;

(iv) suppose that (r-Hs), (r-Convs) and (r-Crit) hold. Then F folds downwards.

Item (i) is a non-symmetric version of the Dolph-Hammerstein Theorem ([18], [23]). In items (ii) and (iii), we circumvent the arguments in [2] and [8] which, under smoothness hypotheses, identify the critical points of $F$ as being folds, a fact which follows from the theorem. For Nemitskii maps $P(u)=f(u)$, we obtain finer results (Theorem 5), from which Theorem 2 follows. Theorem 3 admits variations, but we are interested in displaying a set of techniques, more than specific results. Theorem 3 (iv), for example, is extended in Section 2.8.

Here is a sketch of proof. From (r-H) or (r-Hs), linearizations (or Jacobians) belong to $\mathscr{P}^{b}$. We begin with a perturbation result: for $H \in \mathscr{P}^{b}$ and a special operator $T \in \mathcal{B}(Y), H T$ is also r-special. We use an extension of the Krein-Rutman theorem for cones with possibly empty interior ([17]; for an alternative approach, [9]). It is a diluted version of Theorem 10 in the Appendix, a condensation of results by Marek, Bonsall, Schaefer and Sawashima ([26]).

The convex set $\mathscr{P}^{b}$ has good compactness properties, implying a uniform coercive bound for operators $I-H T$. An argument with covering spaces, the Banach-Mazur theorem, then converts $F$ into adapted coordinates (Proposition 4) $F^{a}: Y \rightarrow Y$ for $F$, a nonlinear rank one perturbation of the identity, already present in the original proof of Theorem 1 by Berger and Podolak ([8]) and in the proof of Theorem 2 in [36]. At this point, the proof of Theorem 3(ii) is at hand.

In combination with the convexity-like hypothesis ( $\mathrm{r}$-Conv), no point in the counterdomain has more than two preimages under $F$ (Proposition 6), so that $F^{a}$ and $F: Y \rightarrow Y$ are simple maps (Theorem 3(ii)). In Theorem 1, the convexity of $f$ is essentially necessary for $F(u)=-\Delta u-f(u)$ to be a fold $([14])$ : the search for further examples led us to the material in this text.

From the adapted coordinates, the inverse under $F$ of a vertical line $L$ is a fiber $\Lambda_{y}$ : our geometric description of $F$ is complete once we compute the asymptotic behavior of $F$ at endpoints of the fiber, informally, how $F\left(\Lambda_{y}\right)$ goes up and down along $L$. Here the arguments bifurcate. For Nemitskii maps, a standard argument (Theorem 5) provides our proof to Theorem 2. For more general $C^{1}$ maps, the existence of one critical point of $F$ (hypothesis (m-Crit)) yields a full fledged critical set (Corollary 2), from which the properness of $F$ follows (Proposition 10 ), and the asymptotic behavior is restricted. Finally, the fact that the images of fibers fold downwards is a consequence of degree theory (Proposition 13). 
Inspired by the fact that Theorem 1 holds for Sobolev and Hölder spaces, we present a set up in Section 2.8 where folds transfer between scales of spaces.

In Section 3, we consider additive, nonlinear perturbations $F(u)=L u-P(u)$ of a self-adjoint operator $L: D \subset H \rightarrow H$ on Hilbert spaces $H$ of functions, possibly defined on unbounded domains. We concentrate on the smallest eigenvalue $\lambda_{1}$ of $L$, which we require to be basic, an isolated point in $\sigma(L)$, whose invariant subspace is generated by a positive eigenfunction $\phi>0$. As in Theorem 1 , the corresponding Theorem 8 does not restrict the analogous parameter $b$.

Again, the appropriate perturbation theory, from Faris and Simon ([21], [28]), delimits a set containing linearizations and Jacobians. The estimates are tighter, being of a spectral nature, and allow for a different technique to derive properness of $F$, which we apply to spaces of functions on unbounded sets. The compactness of resolvents $(L-\gamma I)^{-1}$ is not needed.

We complete the text with some examples in Section 3.4.

Acknowledgements: Calanchi is partially supported by INdAM-GNAMPA Project 2018. Tomei is supported by CAPES, CNPq and FAPERJ. He thanks Boyan Sirakov for a number of illuminating conversations.

\section{Proof of Theorem 3}

\subsection{Cones and the spectral radius of r-special operators}

We recall some basic facts about cones ([17], [26], [22]). Let $Y$ be a real Banach space. We denote its norm by $|\cdot|$. A cone $K \subset Y$ is a closed set for which

$$
0 \in K, \quad K+K \subset K, \quad t \geq 0 \Rightarrow t K \subset K, \quad K \cap(-K)=\{0\} .
$$

It induces a partial order in $Y: y \leq z \Leftrightarrow z-y \in K$. A cone $K$ is reproducing if $K-K=Y$. A cone is normal if, for some $\alpha \in \mathbb{R}, 0 \leq y \leq z \Longrightarrow|y| \leq \alpha|z|$. For a reproducing cone $K$, the dual cone is $K^{*}=\left\{k^{*} \in Y^{*} \mid\left\langle k^{*}, k\right\rangle \geq 0, \forall k \in K\right\}$ (brackets denote the coupling between $Y$ and $Y^{*}$ ). According to a result of Krein ([22], [26]), $K$ is a normal, reproducing cone if and only if $K^{*}$ also is.

An element $k \in K$ is quasi-interior if $\left\langle k^{*}, k\right\rangle \neq 0$, for any $k^{*} \in K^{*} \backslash\{0\}$. Similarly, a linear functional $k^{*} \in K^{*}$ is strictly positive if $\left\langle k^{*}, k\right\rangle>0$ for all $k \in K \backslash\{0\}$. To simplify notation, we denote a quasi-interior point $k$ by $k>0$ and a strictly positive functional $k^{*}$ by $k^{*}>0$.

A cone $K$ is unflattened if, for some $M>0$ and any $y \in Y$, there are $k_{1}, k_{2} \in K$ such that $y=k_{1}-k_{2},\left|k_{1}\right|,\left|k_{2}\right| \leq M|y|$. A reproducing cone is unflattened (a consequence of the Krein-Smulian theorem [40]).

Denote by $\mathcal{B}(Y)$ is the space of bounded linear operators from $Y$ to $Y$ equipped with the sup norm. As usual, the spectral properties of an operator $S \in \mathcal{B}(Y)$ refer to its complexification. Let $r(S)$ be the spectral radius of $S \in \mathcal{B}(Y)$. 
An eigenvalue $\lambda \in \sigma(S)$ of $S \in \mathcal{B}(Y)$ is basic if the following properties hold.

(b-1) There are associated eigenvectors $\phi>0$ of $S, \phi^{*}>0$ of $S^{*}$.

(b-2) Eigenvectors of $S$ (resp. $S^{*}$ ) associated with $\lambda$ are multiples of $\phi$ (resp. $\left.\phi^{*}\right)$.

(b-3) No $y \in Y$ satisfies $(S-\lambda) y=\phi$.

An eigenvalue $\lambda \in \sigma(S)$ satisfying properties (b-2) and (b-3) above is simple. A simple eigenvalue of $S$ is also of $S^{*}$.

An operator $S \in \mathcal{B}(Y)$ is positive $(S \geq 0)$ with respect to a cone $K$ if $S K \subset K$; $S$ is strictly positive $(S>0)$ if $S(K \backslash\{0\}) \subset K \backslash\{0\}$, and $S$ is ergodic if for any $k \in K \backslash\{0\}, S k$ is a quasi-interior element of $K, S k>0$. An operator $S \in \mathcal{B}(Y)$ is $r$-special if it is compact and ergodic with $r(S)>0$. If $S$ is r-special, $S^{*} \in \mathcal{B}\left(Y^{*}\right)$ also is (recall that $K^{*}$ is also a normal, reproducing cone).

The result below is a special case of Theorem 10 in the Appendix.

Theorem 4. Let $K$ be a normal reproducing cone of a real Banach space $Y$. Then an $r$-special operator $S \in \mathcal{B}(Y)$ has a basic eigenvalue $r(S)=r\left(S^{*}\right)$. Eigenvectors of $S$ in $K$ are multiples of $\phi$. For $\mu>r(S),(\mu I-S)^{-1} K \subset K$. If $\mu \in$ $\sigma(S) \backslash\{r(S)\},|\mu|<r(S)$.

We list simple properties of r-special operators frequently used in the text.

Corollary 1. Let $S \in \mathcal{B}(Y)$ be an r-special operator with basic eigenvalue $r(S)$ and associated eigenvectors $\phi>0$ and $\phi^{*}>0$ of $S$ and $S^{*}$. Then the operator $S-r(S) I: Y \rightarrow Y$ is a Fredholm operator of index 0 , the subspaces $V=\langle\phi\rangle$ and $W=\operatorname{Ker} \phi^{*}=\operatorname{Ran}(S-r(S) I)$ are invariant under $S$ and $Y=W \oplus V$.

Proof: A simple consequence of $S$ being r-special.

The basic eigenvalue $r(S)=r\left(S^{*}\right)$ of $S$ and $S^{*}$ and related positive, normalized eigenvectors $\phi, \phi^{*}$, vary smoothly with $S$. We collect some known formulas (Proposition 79.15 in [43], Theorem 4.3 in [26]).

Lemma 1. Let $S \in \mathcal{B}(Y)$ be an r-special operator. There is an open neighborhood $\mathcal{U}$ of $S$ such that, for $\hat{S} \in \mathcal{U}$ the spectral radius $r(\hat{S})$ is a simple eigenvalue. The map $\hat{S} \in \mathcal{U} \mapsto r(\hat{S})$ is real analytic. For appropriate local normalizations, the eigenfunctions $\phi(\hat{S})$ and $\phi^{*}\left(\hat{S}^{*}\right)$ are real analytic maps. For a normalization such that $\left\langle\phi^{*}(\hat{S}), \phi(\hat{S})\right\rangle=1$,

$$
\operatorname{Dr}(\hat{S}) \cdot \bar{S}=\left\langle\phi^{*}(\hat{S}), \bar{S} \phi(\hat{S})\right\rangle
$$

Let $S_{\star} \in \mathcal{B}(Y)$ be r-special. If $S_{\star} \geq S$, then $r\left(S_{\star}\right) \geq r(S)$. Similarly, if $S_{\star}>S$, then $r\left(S_{\star}\right)>r(S)$. 


\subsection{Spectral theory in $\mathscr{P}^{b}$}

Let $T \in \mathcal{B}(Y)$ be an r-special operator with $r(T)=r\left(T^{*}\right)=1$ and associated normal eigenvectors $\phi=\phi(T)>0$ and $\phi^{*}=\phi\left(T^{*}\right)>0$ as in Corollary 1 .

Proposition 1. For $H \in \mathscr{P}^{b}$, the operator $H T \in \mathcal{B}(Y)$ is r-special, with a basic eigenvalue $r(H T)=r\left((H T)^{*}\right)$ bounded away from zero uniformly in $\mathscr{P}^{b}$.

Proof: Since $S$ is r-special and $H T \geq S$ from $(\mathrm{r}-\mathrm{H}), H T$ is r-special and, by Lemma $1, r(H T) \geq r(S)>0$ uniformly in $\mathscr{P}^{b}$. The statements about basic eigenvalues follow from Theorem 4 and its corollary.

Endow $\mathcal{B}(Y)$ with the weak operator topology: for $H_{k}, H_{\infty} \in \mathcal{B}(Y)$,

$$
H_{k} \stackrel{w}{\longrightarrow} H_{\infty} \quad \Leftrightarrow \quad \forall y \in Y, y^{*} \in Y^{*}, \quad\left\langle y^{*}, H_{k} y\right\rangle \rightarrow\left\langle y^{*}, H_{\infty} y\right\rangle .
$$

Since $K$ and $K^{*}$ are reproducing cones, to show $H_{k} \stackrel{w}{\longrightarrow} H_{\infty}$, it suffices to prove

$$
\lim \left\langle y^{*}, H_{k} y\right\rangle=\left\langle y^{*}, H_{\infty} y\right\rangle \quad \text { for } \quad y \in K, y^{*} \in K^{*} .
$$

For a separable space $Y$, the weak topology metrizable.

Lemma 2. The sets $\mathscr{P}^{b}$ and $\mathscr{P}^{0}$ are convex, compact and sequentially compact in the weak operator topology.

Proof: We obtain compactness in the weak operator topology. Closed balls $\{\|H\| \leq C\} \subset \mathcal{B}(Y)$ are compact and sequentially compact in the weak operator topology for a reflexive, separable Banach space $Y$ ([20], [41]). It suffices to show that $\mathscr{P}^{b}$ is closed in the strong operator topology: for $S \leq H_{k} T \leq T,\left\|H_{k}\right\| \leq R$, if $H_{k} \stackrel{s}{\longrightarrow} H$, the same inequalities hold for $H$.

Spectral objects behave well under weak operator convergence.

Lemma 3. Let $H_{k} \in \mathscr{P}^{b}$ such that $H_{k} \stackrel{w}{\longrightarrow} H_{\infty} \in \mathscr{P}^{b}$. Consider sequences $\left\{y_{k} \in Y,\left|y_{k}\right|=1\right\},\left\{g_{k} \in Y, g_{k} \rightarrow g_{\infty}\right\},\left\{\lambda_{k} \in \mathbb{R}, 0<c<\lambda_{k}<C\right\}$ for which

$$
H_{k} T y_{k}=\lambda_{k} y_{k}+g_{k} .
$$

Then, for some (relabeled) subsequences, $y_{k} \rightarrow y_{\infty} \neq 0, \lambda_{k} \rightarrow \lambda_{\infty}>0$ and $H_{\infty} T y_{\infty}=\lambda_{\infty} y_{\infty}+g_{\infty}$.

Proof: Since $Y$ is separable and reflexive, from the sequential Banach-Alaoglu theorem, there is a subsequence $y_{k} \rightarrow y_{\infty} \in Y$ and, from the bounds on $\left\{\lambda_{k}\right\}$, $\lambda_{k} \rightarrow \lambda_{\infty} \neq 0$. As $T$ is compact, for yet another subsequence $y_{k} \rightarrow y_{\infty} \in Y$, $T y_{k} \rightarrow T y_{\infty}$. Take $\ell \in Y^{*}$ and use $H_{k} \stackrel{w}{\longrightarrow} H_{\infty}$ :

$$
\left\langle\ell, H_{\infty} T y_{\infty}\right\rangle=\lim \left\langle\ell, H_{k} T y_{\infty}\right\rangle=\lim \left(\left\langle\ell, H_{k} T\left(y_{\infty}-y_{k}\right)\right\rangle+\left\langle\ell, H_{k} T y_{k}\right\rangle\right) .
$$


As $T\left(y_{k}-y_{\infty}\right) \rightarrow 0$ and the operators $H_{k}$ are uniformly bounded by $(\mathrm{r}-\mathrm{H})$, the first term goes to zero and then

$$
\left\langle\ell, H_{\infty} T y_{\infty}\right\rangle=\lim \left\langle\ell, H_{k} T y_{k}\right\rangle=\lim \left\langle\ell, \lambda_{k} y_{k}+g_{k}\right\rangle=\left\langle\ell, \lambda_{\infty} y_{\infty}+g_{\infty}\right\rangle,
$$

and thus $H_{\infty} T y_{\infty}=\lambda_{\infty} y_{\infty}+g_{\infty}$. If $y_{\infty}=0$, then $T y_{k} \rightarrow T y_{\infty}=0$ and $g_{\infty}=0$. so that, from $H_{k} T y_{k}=\lambda_{k} y_{k}+g_{k}$, since $\left|\lambda_{k} y_{k}\right|$ is bounded away from zero and $\left\{H_{k}\right\}$ is bounded, we must have $y_{\infty} \neq 0$, a contradiction. Thus $y_{\infty} \neq 0$. Finally, $y_{k}=\left(H_{k} T y_{k}-g_{k}\right) / \lambda_{k}$ and the right hand side converges: $y_{k} \rightarrow y_{\infty}$.

Set $V=\langle\phi(T)\rangle, W=\operatorname{Ker} \phi^{*}(T)$ as in Corollary 1, so that $Y=W \oplus V$. Define the associated projection $\Pi_{W}: Y \rightarrow W$.

Proposition 2. For $a \in(0,1]$, there exists $\mathbf{b}>0$ such that, for $H \in \mathscr{P}^{\mathbf{b}}$, the following properties hold.

(i) If $1 \in \sigma(H T)$ is an eigenvalue, then $1=r(H T)$.

(ii) If $(I-H T) w=c \phi(T)$ for $w \in W, c \in \mathbb{R}$, then $w=0$.

(iii) HT does not have more than one eigenvalue larger than 1.

(iv) Assume b for which items (i)-(iii) hold. Then the operators $I-\Pi_{W} H T$ : $W \rightarrow W$ are uniformly coercive:

$$
\exists C>0, \forall v \in W, \forall t \in \mathbb{R}, \quad\left|\left(I-\Pi_{W} H T\right) v\right| \geq C|v|,
$$

Proof: All items will be proved by contradiction.

(i) Suppose $H_{k} \in \mathscr{P}^{b_{k}}, b_{k}<1 / k$ such that $1 \in \sigma\left(H_{k} T\right)$ and $r\left(H_{k} T\right)>1$. As $T \in \mathcal{B}(Y)$ is compact, so is $H_{k} T$, and thus $\sigma\left(H_{k} T\right) \backslash\{0\}$ contains only eigenvalues: there are eigenvectors (normalized in $Y$ ) for which $H_{k} T \psi_{k}=\psi_{k}$ and $H_{k} T \phi_{k}=$ $r\left(H_{k} T\right) \phi_{k}$, such that $r\left(H_{k} T\right)>1$ is a basic eigenvalue, $\phi_{k}>0$. From Lemma 2, up to subsequences, $H_{k} \stackrel{w}{\longrightarrow} H_{\infty} \in \mathscr{P}^{0}$. From Lemma 3, setting $\lambda_{k}=1$ and $g_{k}=0$, there exists $\psi_{\infty} \in Y$ such that (up to subsequences) $\psi_{k} \rightarrow \psi_{\infty} \neq 0$, with $H_{\infty} T \psi_{\infty}=\psi_{\infty}$.

From the monotonicity of the spectral radius (Lemma 1), $1 \leq r\left(H_{k} T\right) \leq$ $\left(1+b_{k}\right) r(T) \rightarrow 1$ and, again by Lemma 3 with $\lambda_{k}=r\left(H_{k} T\right)$, we have $\phi_{k} \rightarrow \phi_{\infty} \neq 0$ in $Y$, with $H_{\infty} T \phi_{\infty}=\phi_{\infty} \in X$.

We show $\phi_{\infty} \neq \pm \psi_{\infty}$. Indeed, there is a sequence of dual normalized eigenvectors $\phi_{k}^{*} \in Y^{*}, \phi_{k}^{*}>0,\left(H_{k} T\right)^{*} \phi_{k}^{*}=\lambda_{k} \phi_{k}^{*}$, which converges to $\phi_{\infty}^{*} \in Y^{*}$ by an argument as in Lemma 3. Also, $\left(H_{\infty} T\right)^{*} \phi_{\infty}^{*}=\phi_{\infty}^{*}, \quad \phi_{\infty}^{*} \in K^{*}, \phi_{\infty}^{*} \neq 0$. From Corollary 1 (i), $\left\langle\psi_{k}, \phi_{k}^{*}\right\rangle=0$ and by taking limits, $\left\langle\psi_{\infty}, \phi_{\infty}^{*}\right\rangle=0$. If $\phi_{\infty}= \pm \psi_{\infty}$, then $\left\langle\psi_{\infty}, \phi_{\infty}^{*}\right\rangle \neq 0$ by positivity. But then $\phi_{\infty}$ and $\psi_{\infty}$ are independent eigenvectors, contradicting the simplicity of $r\left(H_{\infty} T\right)$ (Proposition 1). 
(ii) Take b such that item (i) holds. Write

$$
H_{k} T w_{k}=w_{k}-c_{k} \phi(T), \quad w_{k} \in W \backslash\{0\}, \quad b_{k}=\left\|B_{k}\right\| \rightarrow 0 .
$$

If $c_{k}=0$, one has $1 \in \sigma\left(H_{k} T\right)$ and, from (i), $r\left(H_{k} T\right)=1$. From Proposition 1 , we assume $w_{k}>0$. By positivity, this contradicts $w_{k} \in W=\operatorname{Ker} \phi\left(T^{*}\right)$. Thus $c_{k} \neq 0$ : rescale and consider $c_{k}=1$ for all $k$.

If $\left|w_{k}\right|$ is unbounded, normalize $\hat{w}_{k}=w_{k} /\left|w_{k}\right|$. For a subsequence of $\left\{H_{k}\right\}$, $H_{k} \stackrel{w}{\longrightarrow} H_{\infty} \in \mathscr{P}^{b}$ and, from Lemma $3, \hat{w}_{k} \rightarrow \hat{w}_{\infty} \in W \backslash\{0\}$ and $H_{\infty} T \hat{w}_{\infty}=\hat{w}_{\infty}$. Therefore, $1 \in \sigma\left(H_{\infty} T\right)$ and $r\left(H_{\infty} T\right)=1$ by item (i). Again, take $\hat{w}_{\infty}>0$, contradicting $\hat{w}_{\infty} \in W=\operatorname{Ker} \phi\left(T^{*}\right)$.

Suppose instead that $\left|w_{k}\right|$ is bounded. For subsequences, $w_{k} \rightarrow w_{\infty}$ in $Y$, $H_{k} \stackrel{w}{\longrightarrow} H_{\infty}$, and again from Lemma $3, w_{k} \rightarrow w_{\infty} \neq 0$ and $w_{\infty}-H_{\infty} T w_{\infty}=\phi(T)$.

Again by monotonicity, $1<r\left(H_{k} T\right) \leq\left(1+b_{k}\right) r(T) \rightarrow 1$ and $r\left(H_{\infty} T\right) \in[a, 1]$. If $r\left(H_{\infty} T\right)<1, w_{\infty}=\left(I-H_{\infty} T\right)^{-1} \phi$ and by Theorem $4, w_{\infty} \in K$, contradicting $w_{\infty} \in W \backslash\{0\}$. If $r\left(H_{\infty} T\right)=1$, we must have $\phi \in \operatorname{Ran}\left(I-H_{\infty} T\right)=\operatorname{ker} \phi\left(\left(H_{\infty} T\right)^{*}\right)$ and again this cannot happen, as $\phi\left(\left(H_{\infty} T\right)^{*}\right)>0$.

(iii) Suppose $\lambda, \mu \in \sigma(H T)$ such that $1<\lambda<\mu$ (recall that the top eigenvalue is simple, and the eigenvalues are isolated). Now consider the operator $H T=$ $H T / \lambda$, with eigenvalues $1 / \lambda<1<\mu / \lambda$. The operator belongs to an appropriate $\mathscr{P}^{b}$ (replace $S$ by $S / \lambda$ ) and thus, if $1 \in \sigma(H T)$, we must have $1=r(H T)$, by (i), contradicting $1<\mu / \lambda$.

(iv) Suppose $\epsilon_{k} \rightarrow 0$ and $v_{k} \in W, t_{k} \in \mathbb{R}$ for which $\left|\left(I-\Pi_{W} H T\right) v_{k}\right|<\epsilon_{k}\left|v_{k}\right|$. Thus $v_{k} \neq 0$ and one may normalize, $z_{k}=v_{k} /\left|v_{k}\right|$, so that $z_{k}-\Pi_{W} H_{k} T z_{k} \rightarrow 0$. By Proposition 1, for a subsequence, $H_{k} \stackrel{w}{\longrightarrow} G_{\infty} \in \mathscr{P}^{b}$. As in Lemma 3, for a subsequence $z_{k} \rightarrow z_{\infty} \in W, z_{\infty}-\Pi_{W} G_{\infty} T z_{\infty}=0$ so that $z_{\infty}-G_{\infty} T z_{\infty}=c \phi(T)$.

By item (ii), since $z_{\infty} \in W$, we must have $c=0$ and then $z_{\infty}=0$. On the other hand, since $z_{k} \rightarrow z_{\infty}=0$ and the $H_{k}$ 's are uniformly bounded,

$$
\left|H_{k} T z_{k}\right| \rightarrow 0, \quad \text { and } \quad\left|\Pi_{W} H_{k} T z_{k}\right| \rightarrow 0,
$$

yielding a contradiction: $z_{k}-\Pi_{W} H_{k} T z_{k} \rightarrow 0$ and $\left|z_{k}\right|=1$.

\subsection{Hypothesis ( $\mathrm{r}-\mathrm{H})$ : adapted coordinates}

Let $T \in \mathcal{B}(Y)$ as in Theorem 3, basic eigenvalue $r(T)$ and associated eigenfunctions $\phi=\phi(T) \in Y, \phi^{*}=\phi\left(T^{*}\right) \in Y^{*}$. From Corollary 1, the decomposition $Y=W \oplus V$ for $V=\langle\phi\rangle$ and $W=\operatorname{Ker} \phi^{*}$ induces projections $\Pi_{W}: Y \rightarrow W$ and $\Pi_{V}: Y \rightarrow V$. The horizontal hyperplane at height $t$ is $W^{t}=W+t \phi$. Define the projected restrictions of $F$,

$$
F^{t}: W \rightarrow W, \quad F^{t}(w)=w-\Pi_{W} P(T(w+t \phi)) .
$$

The next proposition finesses some elliptic estimates employed in Theorem 2. 
Proposition 3. Assume the hypotheses of Theorem 3 and $(r-H)$ for $\mathbf{b}$ such that Proposition 2 holds. Then $F^{t}: W \rightarrow W$ are uniformly Lipschitz coercive:

$$
\exists C>0: \quad \forall w, v \in W, \forall t \in \mathbb{R}, \quad\left|F^{t}(w)-F^{t}(v)\right| \geq C|w-v|,
$$

and so are the maps $\Psi: W \oplus \mathbb{R} \rightarrow W \oplus \mathbb{R},(w, t) \mapsto(z, t)=\left(F^{t}(w), t\right)$.

Proof: Suppose by contradiction $\epsilon_{k} \rightarrow 0$ and $w_{k}, v_{k} \in W, t_{k} \in \mathbb{R}$ for which

$$
\left|F^{t_{k}}\left(w_{k}\right)-F^{t_{k}}\left(v_{k}\right)\right|<\epsilon_{k}\left|w_{k}-v_{k}\right| \text {. }
$$

For the linearization $G_{k}=G_{k}\left(T\left(w_{k}+t_{k} \phi\right), T\left(v_{k}+t_{k} \phi\right)\right) \in \mathscr{P}^{b}$,

$$
\begin{gathered}
\left|F^{t_{k}}\left(w_{k}\right)-F^{t_{k}}\left(v_{k}\right)\right|=\left|\left(w_{k}-v_{k}\right)-\Pi_{W}\left(P\left(T\left(w_{k}+t_{k} \phi\right)\right)-P\left(T\left(v_{k}+t_{k} \phi\right)\right)\right)\right| \\
=\left|\left(w_{k}-v_{k}\right)-\Pi_{W} G_{k} T\left(w_{k}-v_{k}\right)\right|>C\left|w_{k}-v_{k}\right| .
\end{gathered}
$$

for the uniform Lipschitz bound $C$ in Proposition 2(iv).

As for $\Psi$, use the equivalent norm ||$|u|||=|||w+t \phi|||=|w|+|t|$.

For the rest of Section 2, $b=\mathbf{b}$ is taken as in Proposition 2. Indeed, this is the required $b>0$ in Theorem 3 .

We recall two well known facts. Let $Z$ be a real Banach space and $U \subset Z$ be an open subset. A continuous function $f: U \rightarrow Z$ is compact if the image of bounded sets is relatively compact.

Theorem (Banach-Mazur, (5.1.4) in [6]). A continuous map $H: Z \rightarrow Z$ is a homeomorphism if and only if it is proper and a local homeomorphism.

Theorem (Schauder Invariance of Domain, [35]). Let $H: U \rightarrow Z$ be a continuous injective function of the form $H(x)=x-Q(x)$ for a compact map $Q: U \rightarrow Z$. Then $H(U) \subset B$ is an open set.

We introduce adapted coordinates ([8], [12]).

Proposition 4. Assume the hypotheses of Proposition 3. For $V \simeq \mathbb{R}$, the map

$$
\Psi: Y=W \oplus \mathbb{R} \rightarrow Y=W \oplus \mathbb{R},(w, t) \mapsto(z, t)=\left(F^{t}(w), t\right)
$$

is a homeomorphism. For $F^{a}(z, t)=F \circ \Psi^{-1}$, the following diagram commutes.

$$
\begin{array}{ccc}
(w, t) & \stackrel{F}{\longrightarrow} & \left(F^{t}(w), \Pi_{V} F(w+t \phi)\right) \\
\Psi^{-1} \nwarrow & & \nearrow F^{a}(z, t)=\left(z, h^{a}(z, t)\right)=\left(z, \Pi_{V} F\left(\Psi^{-1}(z, t)\right)\right)
\end{array}
$$


Proof: Write $y=w+t \phi=\Pi_{W} y+t \phi$. We apply the Banach-Mazur theorem to

$$
\Psi(w, t)=F^{t}(w)+t \phi=(y-t \phi)-\Pi_{W} P(T y)+t \phi \equiv y-Q(y) .
$$

The properness of $\Psi$ follows from Proposition 3. Indeed, given sequences such that $\Psi\left(w_{k}, t_{k}\right)=\left(z_{k}, t_{k}\right) \rightarrow\left(z_{\infty}, t_{\infty}\right)$, use the equivalent norm ||$|y|||=|w|+|t|$,

$$
\left|w_{n}-w_{m}\right|+\left|t_{n}-t_{m}\right|=\left|\Psi\left(w_{n}, t_{n}\right)-\Psi\left(w_{m}, t_{m}\right)\right| \geq C\left(\left|w_{n}-w_{m}\right|+\left|t_{n}-t_{m}\right|\right),
$$

to conclude that the sequences $\left\{\left(z_{k}, t_{k}\right)\right\}$ and $\left\{\left(w_{k}, t_{k}\right)\right\}$ are both Cauchy sequences, necessarily convergent. The same estimate implies the injectivity of $\Psi$. We verify local surjectivity: the continuity of the inverse again would follow from Proposition 3. By the Banach-Mazur theorem, it suffices to show that $\Psi$ is an open map, which implies local surjectivity: we use Schauder's theorem.

We show that, around each $(w, t) \in W \oplus V$, there is a closed ball $D \subset Y$ whose image $Q(D) \subset Y$ is relatively compact for $Q(y)=\Pi_{W} P(T y)$. Since $T$ is compact, and $P$ continuous (Lipschitz), $P \circ T$ is a compact map, and the linear, bounded operators $\Pi_{W}$ preserves compactness.

Thus $\Psi$ is a genuine change of variables. The diagram now is immediate.

Thus, for each $t \in \mathbb{R}$ and $z \in W$, there is a unique $w(z) \in W$ for which $F^{t}(w(z))=z$. Said differently, the image under $F$ of a horizontal hyperplane $W^{t}$ intercepts the vertical line $L_{z}=\{z+h \phi\}$ at a single point: each hyperplane $W^{t}$ contains a unique preimage of $L_{z}$. We denote by $\Lambda_{z}=F^{-1}\left(L_{z}\right)$ the fiber associated with $z$. A fiber contains a unique point on each $W^{t}$ and is parameterized by $\mathbb{R}$ : $\Lambda_{z}(t)=\{y(t)=w(z, t)+t \phi, t \in \mathbb{R}\}$.

The map $\Psi^{-1}$ takes each $W^{t}$ to itself and vertical lines are taken to fibers. The function $t \mapsto h(z, t)=\Pi_{V} F(y(t))=\left\langle\phi\left(T^{*}\right), F(y(t))\right\rangle$ takes the point at height $t$ in the fiber $\Lambda_{z}$ to the point at height $h(z, t)$ of the vertical line $L_{z}$. Equivalently, $h^{a}(z, \cdot)$ takes $(z, t)$ to a point at height $h(z, t)$ of the vertical line $L_{z}$.

Remark For a given $z \in Y$, the solutions of $F(y)=z$ lie in the fiber $\Lambda_{z}$ which, in principle, may be searched by a (one dimensional) continuation method ([37]). Numerical algorithms have been written exploiting this special feature ([12]).

Proposition 5. Under the hypotheses of Theorem 3 (i), F:Y $\rightarrow Y$ is injective.

Proof: For $y_{1}, y_{2} \in Y$ for which $F\left(y_{1}\right)-F\left(y_{2}\right)=0$, use linearizations,

$$
F\left(y_{1}\right)-F\left(y_{2}\right)=\left(y_{1}-y_{2}\right)-G\left(T y_{1}, T y_{2}\right) T\left(y_{1}-y_{2}\right)=0 .
$$

From Proposition 1, $G\left(T y_{1}, T y_{2}\right) \in \mathscr{P}^{b}$, so that $1 \in \sigma\left(G\left(T y_{1}, T y_{2}\right) T\right)$ and thus $r\left(y_{1}, y_{2}\right)=1$, by Proposition 2, contradicting the hypothesis.

Proof of Theorem 3 (i): From Proposition 5, F is injective. Local surjectivity is again a consequence of Schauder's theorem, as in the proof of Theorem 4. We consider the continuity of the (local) inverse. In the notation of Proposition 4, it suffices to show that $F^{a}: W \oplus \mathbb{R} \rightarrow W \oplus \mathbb{R},(z, t) \mapsto\left(z, h^{a}(z, t)\right)$ is a local homeomorphism. The exercise in real analysis is left to the reader. 


\subsection{Hypothesis (r-Conv): simple maps}

As in Theorem 3(ii), suppose (r-H), (r-Conv) hold and $\mathbf{b}$ is taken such that Proposition 2 is true. Set $r\left(G\left(T y_{1}, T y_{2}\right) T\right) \equiv r\left(y_{1}, y_{2}\right)$ and $r(G(T y, 0) T) \equiv r(y)$.

Proposition 6. If $F\left(y_{1}\right)=F\left(y_{2}\right)$, then trichotomy holds: either $y_{1}>y_{2}$ or $y_{1}=y_{2}$ or $y_{1}<y_{2}$. For $y \in Y$, the equation $F\left(y_{1}\right)=y$ has at most two solutions.

Proof: As in Proposition 5, if $F\left(y_{1}\right)-F\left(y_{2}\right)=0, G\left(T y_{1}, T y_{2}\right) \in \mathscr{P}^{b}$ and $r\left(y_{1}, y_{2}\right)=1$. From Theorem 4 , for a choice of sign, $\pm\left(y_{1}-y_{2}\right)$ is a quasi-interior eigenvector of $G\left(T y_{1}, T y_{2}\right) T \in \mathcal{B}(Y)$ : trichotomy holds.

Suppose that $z \in Y$ has three preimages, $z=F\left(y_{1}\right)=F\left(y_{2}\right)=F\left(y_{3}\right)$ for which $y_{1}<y_{2}<y_{3}$. As before, $r\left(y_{3}, y_{2}\right)=r\left(G\left(T y_{2}, T y_{1}\right) T\right)=1$. But from (rConv), since $T y_{1}<T y_{2}<T y_{3}$ (as $T$ is r-special), we must have $G\left(T y_{3}, T y_{2}\right)>$ $G\left(T y_{2}, T y_{1}\right)$ and thus $r\left(y_{3}, y_{2}\right)>r\left(y_{2}, y_{1}\right)$, from the strict monotonicity of the spectral radius (Lemma 1), a contradiction.

If (r-Conv) is not satisfied, the equation $F(y)=z$ for a fixed $z \in Y$ may have a number of solutions, but their graphs still sit one on top of the other.

Proof of Theorem 3 (ii): The homeomorphism $\Psi: W \oplus V \rightarrow W \oplus V$ in Proposition 4 preserves the vertical component: it suffices to prove that $F^{a}$ : $W \oplus V \rightarrow W \oplus V$ is a simple map. This is immediate from Proposition 6: notice that all preimages of a point $z \in Y$ necessarily belong to $F^{a}\left(L_{z}\right)$, where $L_{z} \subset Y$ is the vertical line through $z$.

A finer geometric description of the map $F: Y \rightarrow Y$ follows from the asymptotic behavior of the images of its fibers, as in Theorem 5 below.

\subsection{Nemitskii maps: proof of Theorem 2}

At this point, standard techniques obtain from Theorem 3 (ii) an extension of Theorem 2. We resume the proof of Theorem 3 in Section 2.7.

Let $Y$ be a real, separable, reflexive Banach space of functions containing the constants, for which $K$, the cone of pointwise non-negative functions, is normal and generating. We assume that multiplication $M_{q}$ by bounded functions $q$ : $\mathbb{R} \rightarrow \mathbb{R}$ be bounded, $\left\|M_{q}\right\| \leq \sup |q|$. As in Theorem $3, T \in \mathcal{B}(Y)$ is an r-special operator for which $r(T)=1, T \phi=\phi>0, T^{*} \phi^{*}=\phi^{*}>0,\left\langle\phi^{*}, \phi\right\rangle=1$.

Theorem 5. Let $P(u)=f(u)$ for a strictly convex function $f: \mathbb{R} \rightarrow \mathbb{R}$ such that

$$
0<a=\inf _{r \neq s} q(r, s)<1<1+b=\sup _{r \neq s} q(r, s) .
$$

Then, for some $b>0, F=I-P T: Y \rightarrow Y$ folds downwards with respect to $\phi$. 
Here, the Newton quotient $q(r, s)$ is $q(r, s)=(q(r)-q(s)) /(r-s)$ for $r \neq s$.

Proof: We check the hypotheses of Theorem 3 (ii). As in [2], use linearizations $G(u, v)=M_{q(u, v)}$, where $M_{q(u, v)}$ is the multiplication operator by the bounded function $q(u, v)(x)=q(u(x), v(x))$ for $u(x) \neq v(x)$ and $q(u, v)(x)=a$ otherwise.

Clearly $P, G(u, v): Y \rightarrow Y$ are well defined and continuous, as $f$ is Lipschitz. Moreover, $M_{q(u, v)}=G(u, v) \in \mathscr{P}^{b}$ for $S=a T, R=1+b$. Indeed,

$q(u, v)=\alpha+\beta=\min \{q(u, v), 1\}+(q(u, v)-\min \{q(u, v), 1\}), M_{q(u, v)}=M_{\alpha}+M_{\beta}$, and then $S=a T \leq M_{\alpha} T \leq T,\left\|M_{\beta}\right\| \leq b$. This settles $(\mathrm{r}-\mathrm{H})$.

For (r-Conv), consider pointwise estimates for $x \in \Omega$, the domain of the functions in $Y$ : as $f$ is strictly convex, for $y_{1}, y_{2}, z_{1}, z_{2} \in Y$ as in (m-Conv),

$$
\frac{f\left(y_{1}(x)\right)-f\left(z_{1}(x)\right)}{y_{1}(x)-z_{1}(x)}>\frac{f\left(y_{2}(x)\right)-f\left(z_{2}(x)\right)}{y_{2}(x)-z_{2}(x)} .
$$

Thus $G\left(y_{1}, z_{1}\right)-G\left(y_{2}, z_{2}\right) \in \mathcal{B}(Y)$ is strictly positive. Now use Theorem 3 (ii) to conclude that $F$ is a simple map.

From the hypotheses on $f$, there are lines $\ell_{ \pm}(t)=\alpha_{ \pm} t+\beta_{ \pm}$such that $f(t)>$ $\ell_{ \pm}(t)$ with $\alpha_{-}<1=r(T)<\alpha_{+}$([36], for example).

We compute the height of the images of the fiber $y(t)=w(t)+t \phi, w(t) \in W$ :

$$
\begin{aligned}
h(F(y(t))) & =\left\langle\phi^{*}, F(y(t))\right\rangle=\left\langle\phi^{*}, w(t)+t \phi-P(T y(t))\right\rangle=t-\left\langle\phi^{*}, f(T y(t))\right\rangle . \\
& <t-\left\langle\phi^{*}, \alpha_{+} T(w(t)+t \phi)+\beta_{+}\right\rangle=\left(1-\alpha_{+}\right) t-\left\langle\phi^{*}, \beta_{+}\right\rangle,
\end{aligned}
$$

(recall $\left.\beta_{+} \in Y\right)$. As $t \rightarrow \infty$, we have $h(F(y(t))) \rightarrow-\infty$ since $1-\alpha_{+}<0$. A similar estimate using the line $\ell_{-}$obtains $h(F(y(t))) \rightarrow-\infty$ also for $t \rightarrow-\infty$.

By definition, the image $F(y(t))$ of the fiber $y(t)$ lies in a vertical line of $Y$. As $t \rightarrow \pm \infty, h(F(y(t))) \rightarrow-\infty: F$ folds downwards.

The set $\mathscr{P}^{b}$ is not necessary in this case: the implicit set in the arguments is

$$
\mathscr{Q}^{a b}=\left\{M_{q} \in \mathcal{B}(Y): q \in L^{\infty}(\Omega), a \leq q(x) \leq 1+b \text { a.e. }\right\},
$$

which is convex and sequentially compact with respect to the weak operator topology of $\mathcal{B}(Y)$ from the Banach-Alaoglu Theorem. Clearly, $\mathscr{Q}^{a b} \subset \mathscr{P}^{b}$.

\section{Proof of Theorem 2:}

The space $Y=L^{n}(\Omega)$ clearly satisfies the hypotheses of Theorem 5, as does $K \subset Y$, the cone of nonnegative functions (notice that nonnegative functions in $X=W^{2, n}(\Omega) \cap W_{0}^{1, n}(\Omega)$ do not form a normal cone). Without loss, $\lambda_{1}=\lambda_{1}(\mathbf{L})=$ 0 (simply replace $\mathbf{L}$ by $\mathbf{L}-\lambda_{1}$ ). Denote by $\mathbb{C}^{+}=\{z \in \mathbb{C}, \Re z>0\}$ the open right half-plane: by hypothesis, $\sigma(\mathbf{L}) \subset \mathbb{C}^{+} \cup\{0\}$.

Recall $X=W^{2, n} \cap W_{0}^{1, n} \subset C^{0}$. The operator $\mathbf{L}+\gamma I: X \rightarrow Y$ is invertible for $\gamma>0$. Denote the compact inclusion of $X$ in $Y$ by $\iota: X \rightarrow Y$. Given 
the conformal mapping $z \mapsto \Gamma_{\gamma}(z)=\gamma /(z+\gamma), \gamma>0$, define the operator $T=\iota \circ \gamma(\mathbf{L}+\gamma I)^{-1} \in \mathcal{B}(Y)$. As $\Gamma_{\gamma}$ takes $\mathbb{C}^{+}$to the open disk $D=\{|z-1 / 2|<$ $1 / 2\}, \sigma(T) \subset D \cup\{1\}$ (recall the Dunford-Schwartz functional calculus for closed operators $L: \operatorname{Dom}(L) \rightarrow Y$ [19], p. 599). Also, $\phi_{1}>0$ is an eigenvector of both $\mathbf{L}$ and $T$ for eigenvalues 0 and $r(T)=1$ respectively.

The hypotheses of Theorem 3 for $T$ are satisfied: from well known properties of the operator $\mathbf{L}: X \rightarrow Y([5],[4]), T \in \mathcal{B}(Y)$ is r-special. Also, $r(T)=1, T \phi_{1}=$ $\phi_{1} \in Y, \phi_{1}>0$ and similar hypotheses hold for $\phi_{m}^{*}$, since $T$ is r-special.

The map $\mathbf{P}: Y \rightarrow Y, \mathbf{P}(u)=\mathbf{f}(u)$ is associated with a strictly convex function $\mathbf{f}: \mathbb{R} \rightarrow \mathbb{R}$ for which $\mathbf{a}=\inf q_{\mathbf{f}}(x, y)<0<\mathbf{b}=\sup q_{\mathbf{f}}(x, y)$. Set $P=I+\mathbf{P} / \gamma$, so that $P(y)=f(y)$ for $f=1+\mathbf{f} / \gamma$ and

$$
0<a=1+\mathbf{a} / \gamma<r(\tilde{T})=1<1+b=1+\mathbf{b} / \gamma .
$$

As in the proof of Theorem $5, P$ satisfies $(\mathrm{r}-\mathrm{H})$ and (r-Conv). Now take $\gamma$ so large that $b>0$ complies with the hypothesis of Theorem 5. Thus $F=I-P T$ : $Y \rightarrow Y$ folds downwards, and the same must happen to $\mathbf{F}=\mathbf{L}-\mathbf{P}: X \rightarrow Y$ : Theorem 2 holds for the appropriate $\mathbf{b}=\gamma b$.

We consider extensions of Theorem 2, by replacing $\mathbf{L}$ with an appropriate $g(\mathbf{L})$ which still induces an r-special operators $T$ through the conformal mapping $\Gamma$. We assume $\lambda_{1}=0$.

As a simple example, for $\mathbf{L}$ as above, powers $\mathbf{L}^{k}$, for some $k \in \mathbb{N}$ still yield r-special operators, provided $\sigma\left(\mathbf{L}^{k}\right)$ lies in the closed half-plane. Clearly, this is the case if $\sigma(\mathbf{L}) \subset[0, \infty]$. No other eigenvalue of $\mathbf{L}^{k}$ is sent to zero and thus $T=\Gamma_{\gamma}\left(\mathbf{L}^{k}\right)$ still has a simple spectral radius 1 . Compactness and preservation of the cone $K$ are immediate.

One might consider more complicated functions $g$, at the cost of additional hypothesis (a decay rate of the resolvent of $L$ would be natural). For example, sectorial properties of $\sigma(\mathbf{L})$ might lead to fractional powers for which the associated $T$ is still r-special. For a related situation, see Proposition 18.

\subsection{Consequences of properness: Theorem 3 (iii)}

The function below is already given in adapted coordinates,

$$
(x, y) \mapsto(x,(1-x y) y) .
$$

Vertical lines in the right (resp. left) half-plane fold downwards (resp. upward), the vertical axis stays fixed. This map is not proper. Under properness, the height functions of the image of all vertical lines have the same asymptotic behavior, as shown in the proposition below. (Proposition 10 of [36], in the current notation).

Proposition 7. Let $Z$ be a real Banach space. A proper map $A: Z \oplus \mathbb{R} \rightarrow Z \oplus \mathbb{R}$ of the form $A(z, t)=(z, \alpha(z, t))$ for which no point has more than two preimages is either a homeomorphism or folds vertically. 
Proof of Theorem 3 (iii): Combine Theorem 3 (ii) and Proposition 7.

For a fiber $\Lambda=\{u(t), t \in \mathbb{R}\}$, asymptotic information of the height $h(t)$ of its image $F(u(t))=z+h(t) \phi$ determines if $F$ is a homeomorphism or a fold, as well as the type of the fold. By Proposition 7 , if $F$ is proper, such limits are independent of the fiber. One approach to the asymptotic limits has been used at the end of the proof of Theorem 5: the hypothesis on $f$ is so stringent that the images of fibers and vertical lines in the domain have the same asymptotic behavior. We present an alternative approach in Section 2.7.

\subsection{Hypotheses (r-Hs), (r-Convs) and (r-Crit): smoothness}

When $F$ is a $C^{1}$ map we use different tools. From Proposition 7 , the proof of Theorem 3 (iv) is complete once we establish that $F$ is proper (Proposition 10) and $F$ folds downwards (Proposition 13 (iii)).

Throughout this section, we assume the hypotheses of Theorem 3(iv): (r-Hs), (r-Convs) and (r-Crit) and the usual $\mathbf{b}>0$ for which Proposition 2 holds. For $F(y)=y-P(T y): Y \rightarrow Y$, we have $D F(y) z=z-D P(T y) T$. From (r-Crit), there is $y_{c} \in Y$ for which $D F\left(y_{c}\right)$ is not invertible. Without loss,

$$
y_{c}=0 \quad F(0)=0
$$

(compose $F$ with translations in domain and counterdomain - hypotheses (r-Hs) and (r-Convs) are invariant under these operations).

We take the linearization $G(y, z) \in \mathcal{B}(Y)$ to be

$$
P(y)-P(z)=\int_{0}^{1} D P(z+s(y-z)) d s(y-z)=: G(y, z)(y-z),
$$

so that $G(y, y)=J(y)=D P(y)$. Set $G(T y)=G(T y, 0)$. As $F(0)=0$ we have $P(T y)=G(T y) T y$ with $G(T y) T \in \mathcal{B}(Y)$. Also, $F(y)=y-G(T y) T y$.

Proposition 8. For $y, z \in Y, J(y), G(y, z) \in \mathscr{P}^{b}$. The maps

$$
y, z \mapsto G(y, z) \in \mathcal{B}(Y) \quad \text { and } \quad y, z \mapsto r(G(y, z) T) \in \mathbb{R}
$$

are continuous. The linearizations $G(y, z)$ satisfy $(r-H)$ and (r-Conv).

Proof: From $(\mathrm{r}-\mathrm{Hs}), D P(y), D P(z) \in \mathscr{P}^{b}$. From Lemma 2, convex combinations of Jacobians, as well as integrals along a segment, also belong to $\mathscr{P}^{b}$ and satisfy (r-H) and (r-Conv). The map $y, z, \mapsto G(y, z) \in \mathcal{B}(Y)$ is continuous as $P: Y \rightarrow Y$ is $C^{1}$. Lemma 1 implies the continuity of $r(G(y, z) T)$.

The critical set $\mathcal{C}$ of $F$ consists of the points in $Y$ for which $D F$ is not invertible. From Propositions 1 and 2,

$$
\mathcal{C}=\{y \in Y \mid 0 \in \sigma(D F(y))\}=\{y \in Y \mid r(y)=r(J(T y) T)=1\} .
$$


Our next step is Proposition 9: critical points are abundant. We obtain (exactly) one for each line $\{y+s \psi, t \in \mathbb{R}, \psi>0\}$. Set $B_{\delta}^{Y}=\{z \in Y,|z| \leq \delta\}$.

Lemma 4. Let $y, z, \psi \in Y, \psi>0, \delta>0$. Then there are $s^{*} \in \mathbb{R}, k \in K$ and $z_{\delta} \in B_{\delta}^{Y}$ such that $y+s^{*} \psi=z+z_{\delta}+k$.

Let $z \in L^{2}(0,1)$ not bounded from below, $y=0, \psi \equiv 1$. Then no constant $s$ gives $0<s \psi=z+k$ : the small perturbation $z_{\delta}$ is needed.

Proof: If one cannot obtain $-z+y+s^{*} \psi=k+z_{\delta}$, the line $L=\{-z+y+s \psi, s \in$ $\mathbb{R}\}$ does not intercept $K_{\delta}$, the (closed) convex span of $K$ and $B_{\delta}^{Y}$, a set having $\psi \in K$ in its interior. By the Hahn-Banach theorem, $L$ and $K_{\delta}$ are separated by $H=\{z \in Y,\langle\ell, z\rangle=c\}$, the level $c \in \mathbb{R}$ of a functional $\ell \in Y^{*} \backslash\{0\}$ : $\left.\ell\right|_{L} \leq c \leq\left.\ell\right|_{K_{\delta}}$.

We first show that $\ell \in K^{*} \backslash\{0\}$. Indeed, if there is $k \in K$ such that $\langle\ell, k\rangle<0$, then for large positive $t$ (so that $t k \in K$ ), $\langle\ell, t k\rangle<c$, a contradiction. Also, since $\ell(y+s \psi)=\ell(y)+s \ell(\psi) \leq c$ for all $s \in \mathbb{R}$, we must have $\ell(\psi)=0$. But, as $\ell \in K^{*} \backslash\{0\}$ and $\psi>0$, we must have $\ell(\psi)>0$.

As usual, $T \in \mathcal{B}(Y)$ is r-special, $r(T)=1$. Set $r(y)=r(G(T y) T)$.

Proposition 9. Let $y \in Y, \psi>0$.

(i) The map $s \in \mathbb{R} \rightarrow r(y+s \psi)=r(G(T(y+s \psi), 0) T)$ is strictly increasing. For some $s_{0} \in \mathbb{R}, r\left(y+s_{0} \psi\right)=1$.

(ii) For all increasing sequences $s_{k} \rightarrow \infty$, the operators $\left\{G\left(T s_{k} \psi\right)\right\} \subset \mathscr{P}^{\mathbf{b}}$ have a common weak limit $G\left(T s_{k} \psi\right) \stackrel{w}{\longrightarrow} H_{\infty} \in \mathscr{P}^{\mathbf{b}}$. An analogous result holds for decreasing sequences $s_{k} \rightarrow-\infty$.

Proof: (i): By Proposition 1, $G(T(y+s \psi)) T \in \mathcal{B}(Y)$ is r-special and the eigenvalue $r(y+s \psi)$ is basic. The map $s \mapsto r(y+s \psi)$ is a continuous, strictly increasing function, from Lemma 1. We search for $s_{+}$for which $r\left(y+s_{+} \psi\right)>1$.

From (r-Crit), $0 \in \mathcal{C}$, so that $r(0)=r(G(0,0) T)=1$. By monotonicity (Lemma 1), for $z_{+}=p \psi, p>0$, we have $r\left(z_{+}\right)=r\left(G\left(T z_{+}\right) T\right)>1$. By continuity, there is $\delta>0$ for which $r\left(z_{+}+z_{\delta}\right)>1$ for $z_{\delta} \in B_{\delta}^{Y}$.

Apply Lemma 4 for $z=z_{+}$: we obtain $s^{*} \in \mathbb{R}, k \in K$, and $z_{\delta} \in B_{\delta}^{Y}$ such that $y+s^{*} \psi=z_{+}+k+z_{\delta}$. We compare the spectral radius at different points:

$$
r\left(y+s^{*} \psi\right)=r\left(z_{+}+k+z_{\delta}\right) \geq r\left(z_{+}+z_{\delta}\right)>1 .
$$

Set $s_{+}=s^{*}$. Simple modifications of Lemma 4 obtain $s_{-}$for which $r\left(y+s_{-} \psi\right)<1$. By continuity and monotonicity, there is a single $s_{0}$ such that $r\left(y+s_{0} \psi\right)=1$.

(ii): From (r-Convs), as $\psi>0$, the linearizations $G(T s \psi)$ increase with $s$. Increasing sequences $\left\{s_{k}^{1}\right\}$ and $\left\{s_{k}^{2}\right\}$ yield limits $H_{1}$ and $H_{2}$ by Lemma 2. For $y^{*} \in K^{*}, z \in K$, the sequences $\tau_{k}^{1}=\left\langle y^{*}, G\left(T s_{k}^{1} \psi\right) z\right\rangle$ and $\tau_{k}^{2}=\left\langle y^{*}, G\left(T s_{k}^{2} \psi\right) z\right\rangle$ increase and interlace: for every $N \in \mathbb{N}$, there are $m, n>N$ such that $\tau_{m}^{1}>\tau_{n}^{2}$ and other $m, n>N$ with $\tau_{m}^{1}<\tau_{n}^{2}$. Thus $H_{1}=H_{2}:=H_{\infty}$. 
Corollary 2. Every vertical line $\{w+t \phi(T), t \in \mathbb{R}\}$ in the domain $Y$ of $F$ contains a critical point of $F$. The same holds for lines $\{t \psi, \psi>0, t \in \mathbb{R}\}$.

Proposition 10. $F: Y \rightarrow Y$ is proper.

The argument follows in spirit the proof of the properness of $F$ in [2].

Proof: Let $\left\{y_{k}\right\} \subset Y$ be a sequence for which $F\left(y_{k}\right)=g_{k} \rightarrow g_{\infty} \in Y$. Suppose first that $\left\{y_{k}\right\}$ is bounded in $Y$. Since the linearizations $G(y, z), G(y)$ are uniformly bounded and $P(0)=0$,

$$
\left|P\left(T y_{k}\right)\right|=\left|G\left(T y_{k}\right) y_{k}\right| \leq C\left|y_{k}\right|
$$

is also bounded in $Y$, and the sequence $y_{k}=P\left(T y_{k}\right)+g_{k}$ is bounded. Therefore, for some subsequence, $y_{k} \rightarrow y_{\infty}$. By the compactness of $T$ (and the fact that $P$ is Lipschitz), again up to a subsequence, $T y_{k} \rightarrow T y_{\infty}$ and thus $P\left(T y_{k}\right) \rightarrow P\left(T y_{\infty}\right)$. Therefore, $y_{k} \rightarrow y_{\infty}=P\left(T y_{\infty}\right)+g_{\infty}$.

Consider now $\left\{y_{k}\right\}$ unbounded in $Y$, for strictly increasing $\left|y_{k}\right| \rightarrow \infty$. Normalize $\hat{y}_{k}=y_{k} /\left|y_{k}\right|$ and use the linearization $G_{k}=G\left(T y_{k}\right)$ :

$$
\hat{y}_{k}-P\left(T y_{k}\right) /\left|y_{k}\right|=\hat{y}_{k}-G_{k} \hat{y}_{k}=g_{k} /\left|y_{k}\right| \rightarrow 0 .
$$

As usual, since $G_{k} \in \mathscr{P}^{b}$, up to subsequences, $G_{k} \stackrel{w}{\longrightarrow} G_{\infty}$ for some $G_{\infty} \in \mathscr{P}^{b}$, and from Lemma 3 ,

$$
\hat{y}_{k} \rightarrow \hat{y}_{\infty} \neq 0, \text { and } G_{\infty} T \hat{y}_{\infty}=\hat{y}_{\infty} .
$$

By Propositions 1 and $2, r\left(G_{\infty} T\right)=1$ is a basic eigenvalue and either $\hat{y}_{\infty}$ or $-\hat{y}_{\infty}$ is a quasi-interior point. Say $\hat{y}_{\infty}>0$.

Consider now the projections $y_{k}^{p}=\left|y_{k}\right| \hat{y}_{\infty}$ along the ray $\left\{t \hat{y}_{\infty}, t>0\right\}$. Write the equation $y_{k}-P\left(T y_{k}\right)=g_{k}$ as

$$
y_{k}^{p}-P\left(T y_{k}^{p}\right)=P\left(T y_{k}\right)-P\left(T y_{k}^{p}\right)+g_{k}+y_{k}^{p}-y_{k} .
$$

The right hand side is $o\left(\left|y_{k}\right|\right)$. This is clear for the bounded sequence $\left\{g_{k}\right\}$ and for $\left(y_{k}^{p}-y_{k}\right)=\left|y_{k}\right|\left(\hat{y}_{k}-\hat{y}_{\infty}\right)$, since $\hat{y}_{k} \rightarrow \hat{y}_{\infty} \in Y$. Moreover,

$$
\left|P\left(T y_{k}\right)-P\left(T y_{k}^{p}\right)\right| \leq C\left|y_{k}-y_{k}^{p}\right|=C\left|y_{k}\right|\left|\hat{y}_{k}-\hat{y}_{\infty}\right|=o\left(\left|y_{k}\right|\right) .
$$

Set $P\left(T y_{k}^{p}\right)=G_{k}^{p} T y_{k}^{p}$. By Lemma 2 , for a subsequence, $G_{k}^{p} \stackrel{w}{\longrightarrow} G_{\infty}^{p} \in \mathscr{P}^{b}$.

Divide equation (1) by $\left|y_{k}\right|$ to obtain

$$
\lim \left(y_{k}^{p} /\left|y_{k}\right|-P\left(T y_{k}^{p}\right) /\left|y_{k}\right|\right)=\hat{y}_{\infty}-G_{\infty}^{p} T \hat{y}_{\infty}=0,
$$

and, as $\hat{y}_{\infty}>0,1 \in \sigma\left(G_{\infty}^{p} T\right)$ and, by Theorem $4, r\left(G_{\infty}^{p} T\right)=1$. This contradicts Proposition 9: the points $y_{k}^{p}$ belong to a common ray $\left\{s \hat{u}_{\infty}=s \psi, s \in \mathbb{R}^{+}\right\}$. Combining items (i) and (ii), $r\left(G_{k}^{p} T\right)$ increases and takes a value larger than one: the limit cannot be one.

Minor adjustments handle also the case $\hat{y}_{\infty}<0$. 
When $F: Y \rightarrow Y$ is a $C^{1}$ map, the adapted coordinates in Proposition 4 are obtained from $C^{1}$ changes of variables $\Psi: Y \rightarrow Y$. The inverses of vertical lines $L_{z}=\{z+h \phi, h \in \mathbb{R}\}$ under $F$, the fibers

$$
\Lambda_{z}=\{y(t)=w(z, t)+t \phi, w(z, t) \in W, t \in \mathbb{R}\},
$$

have $C^{1}$ parameterizations. Fix $z \in W$, set $F(y(t))=z+h(t) \phi$, where again $h(\cdot): \mathbb{R} \rightarrow \mathbb{R}$ is a $C^{1}$ map. Let $\lambda(y(t))=1-r(D P(T y(t) T))$.

Lemma 5. For a continuous strictly positive $\alpha: \mathbb{R} \rightarrow \mathbb{R}^{+}, h^{\prime}(t)=\lambda(y(t)) \alpha(t)$.

Part of this lemma is Proposition 2.6 in [14].

Proof: Differentiate $F(y(t))=z+h(t) \phi$ along a fiber $y(t)$ to obtain

$$
D F(y(t)) y^{\prime}(t)=h^{\prime}(t) \phi .
$$

Since $\Pi_{W} D F(y(t)): W \rightarrow W$ is always invertible, at a critical point $y\left(t_{c}\right)$, we must have $h^{\prime}\left(t_{c}\right)=0$ and $\phi \notin D F(y(t))$. Thus $y^{\prime}\left(t_{c}\right)$ is a generator of Ker $D F\left(y\left(t_{c}\right)\right)$, and either $y^{\prime}\left(t_{c}\right)>0$ or $-y^{\prime}\left(t_{c}\right)>0$. As $y^{\prime}\left(t_{c}\right)=w^{\prime}\left(t_{c}\right)+\phi$ and $W=\operatorname{Ker} \phi^{*}$,

$$
\left\langle\phi^{*}, y^{\prime}\left(t_{c}\right)\right\rangle=\left\langle\phi^{*}, w^{\prime}\left(t_{c}\right)+\phi\right\rangle=\left\langle\phi^{*}, \phi\right\rangle>0
$$

and $y^{\prime}\left(t_{c}\right)>0$. Conversely, if $h^{\prime}(\bar{t})=0$, as $y^{\prime}(\bar{t})=w^{\prime}(\bar{t})+\phi \neq 0$, we must have that $D F(y(\bar{t}))$ is not invertible and hence $y(\bar{t})=y_{c}$. Adding up, $y(t)$ is a critical point of $F$ if and only if $h^{\prime}(t)=0$.

As $D F(y(t))=I-H(y(t)) T$ for $H(y(t))=D P(T y(t)) \in \mathscr{P}^{b}$, eigenvectors $\phi(y(t))>0$ and $\phi^{*}(y(t))>0$ are associated with $r(H(y(t)))$. Use brackets for evaluation of dual vectors,

$$
\left\langle\phi^{*}(y(t)), D F(y(t)) y^{\prime}(t)\right\rangle=h^{\prime}(t)\left\langle\phi^{*}(y(t)), \phi\right\rangle
$$

and $h^{\prime}(t)\left\langle\phi^{*}(y(t)), \phi\right\rangle=\left\langle D F(y(t))^{*} \phi^{*}(y(t)), y^{\prime}(t)\right\rangle=\lambda(y(t))\left\langle\phi^{*}(y(t)), y^{\prime}(t)\right\rangle$.

As $\left\langle\phi^{*}(y(t)), \phi\right\rangle>0,\left\langle\phi^{*}(y(t)), y^{\prime}(t)\right\rangle=0$ if and only if $h^{\prime}(t)=0$. From the first part of the proof, $t=t_{c}$ for a critical point $y\left(t_{c}\right)$ and then $\left\langle\phi^{*}\left(y\left(t_{c}\right)\right), y^{\prime}\left(t_{c}\right)\right\rangle=0$, which cannot happen because $y^{\prime}\left(t_{c}\right)>0$. Thus $\left\langle\phi^{*}(y(t)), y^{\prime}(t)\right\rangle>0$ and the quotient $\alpha(t)=\left\langle\phi^{*}(y(t)), y^{\prime}(t)\right\rangle /\left\langle\phi^{*}(y(t)), \phi\right\rangle$ is strictly positive.

Proposition 11. Every fiber contains points $y^{-}, y^{0}$ and $y^{+}$for which $r\left(y^{-}\right)<$ $r\left(y^{0}\right)=1<r\left(y^{+}\right)$, or equivalently, $\lambda\left(y^{-}\right)>\lambda\left(y^{0}\right)=0>\lambda\left(y^{+}\right)$.

Proof: Let $z^{0} \in Y$ be critical. For $\psi>0$, set $z^{ \pm}=z^{0}+s^{ \pm} \psi$, where $s^{-}<0<s^{+}$. By monotonicity (Lemma 1), $r\left(z^{-}\right)<r\left(z^{0}\right)=1<r\left(z^{+}\right)$. From Theorem 3 (ii), the height function $h(t)$ of each fiber is either strictly increasing or unimodal. From properness (Proposition 10), all functions $h(t)$ have the same asymptotic behavior (Proposition 7). 
If $h$ along a fiber is strictly decreasing (hence along all fibers), for example, we must have $h^{\prime}(t) \leq 0$. But from Proposition $5, \lambda(y(t)) \leq 0$, or $r(y(t)) \geq 1$, and this violates the existence of a point $z^{-}$for which $r\left(z^{-}\right)<1$. Similarly $h$ cannot be strictly increasing. We are left with folds, implying the result.

We extend Proposition 6. A point $y \in Y$ is regular if it is not critical.

Proposition 12. Let $y_{c} \in Y$ be a critical point of $F, r\left(y_{c}, y_{c}\right)=r\left(J\left(T y_{c}\right) T\right)=1$. Then $F\left(y_{c}\right)$ has a single preimage (clearly $y_{c}$ itself). For a regular point $y \in Y$, the point $F(y)$ only has regular preimages.

Proof: Suppose $F(y)=F\left(y_{c}\right)$, with $y \neq y_{c}$. Then, as in Proposition 6, trichotomy holds: $y>y_{c}$, or $y<y_{c}$ and $r\left(y, y_{c}\right)=1$. Since $T$ is ergodic (rspecial), either $T y>T y_{c}$ or $T y<T y_{c}$. Since by hypothesis $r\left(y_{c}, y_{c}\right)=1$, from (r-Convs), in both cases $r\left(y, y_{c}\right) \neq 1$. The second statement now is obvious.

From Proposition 10, the map $F=I-P T: Y \rightarrow Y$ is proper and clearly $P T: Y \rightarrow Y$ is a compact map. Moreover, any point has at most two preimages (Proposition 6). The degree $\operatorname{deg}(F)$ is a well defined map, and we compute it by considering indices at regular points. More explicitly, for appropriate $y \in Y$, set $\operatorname{ind}(F, y)=\operatorname{deg}\left(F, B_{\epsilon}(y), F(y)\right)$, for small $\epsilon>0$.

Proposition 13. (i) For $\lambda(y)=1-r(y)$, $\operatorname{ind}(F, y)=\operatorname{sgn} \lambda(y)$.

(ii) The function $F: Y \rightarrow Y$ is not injective. Also, $\operatorname{deg}(F)=0$.

(iii) The function $F: Y \rightarrow Y$ folds downwards.

Proof: (i) From a standard argument using the inverse function theorem, at a regular point $y \in Y$, ind $(F, y)=\operatorname{ind}\left(F^{L}, y\right)$, where

$$
F^{L}(z)=F(y)+D F(y)(z-y)=F(y)+(z-y)-J(T y) T(z-y) .
$$

Recall that, for a compact operator $K$, the degree of $I-K \in \mathcal{B}(Y)$ is $\operatorname{par}(K)$, the parity of the number of strictly negative eigenvalues of $I-K$, or equivalently, the parity of the number of eigenvalues of $K$ which are larger than 1 . Thus, for the restricted affine map $F^{L}, \operatorname{deg}\left(F^{L}, B_{\epsilon}(y), F^{L}(y)\right)=\operatorname{par}(J(T y) T)$. By Proposition 2, $J(T y) T$ has zero or one eigenvalue larger than 1 , depending if $r(y, y)<1$ or $r(y, y)>1$ and then $\operatorname{ind}(F, y)=\operatorname{par}(J(T y) T)=1$ or -1 , always $\operatorname{sgn} \lambda(y)$.

(ii) Let $y_{c} \in Y$ be critical. For $\psi>0$, set $y^{ \pm}=y_{c}+s^{ \pm} \psi$, where $s^{-}<0<s^{+}$. By monotonicity (Lemma 1 ), the sign of $s^{ \pm}$equals the sign of $1-r\left(J\left(T y^{ \pm}\right) T\right.$ ), so that $\operatorname{ind}\left(F, y^{-}\right)=1$, and ind $\left(F, y^{+}\right)=-1$. By Propositions 6 and 12 , the image $F(y)$ of any regular point $y \in Y$ has at most two regular preimages, one being $y$. Call the preimages of $F\left(y^{ \pm}\right)$by $y_{1}^{ \pm}$and $y_{2}^{ \pm}$, where, say, $y_{1}^{ \pm}=y^{ \pm}$. Then

$$
\operatorname{deg}(F)=\operatorname{ind}\left(F, y_{1}^{-}\right)+\operatorname{ind}\left(F, y_{2}^{-}\right)=\operatorname{ind}\left(F, y_{1}^{+}\right)+\operatorname{ind}\left(F, y_{2}^{+}\right)
$$




$$
=1+\operatorname{ind}\left(F, y_{2}^{-}\right)=\operatorname{ind}\left(F, y_{2}^{+}\right)-1,
$$

and each unknown index is either 1 or -1 . Then necessarily

$$
\operatorname{deg}(F)=0, \quad \operatorname{ind}\left(F, y_{2}^{-}\right)=-1, \quad \operatorname{ind}\left(F, y_{2}^{+}\right)=1 .
$$

(iii) Since $F$ is not injective, consider $y^{-} \neq y^{+}$such that $F\left(y^{+}\right)=F\left(y^{-}\right)$. Both points are regular (Proposition 12) and clearly belong to the same fiber, and we write $y^{+}=y\left(t^{+}\right)=w^{+}+t^{+} \phi, y^{-}=y\left(t^{-}\right)=w^{-}+t^{-} \phi$, with $t^{+}>t^{-}$. From (ii), we must have that $\operatorname{ind}\left(F, y^{+}\right)$and $\operatorname{ind}\left(F, y^{-}\right)$are opposite, so that $\operatorname{deg}(F)=0$, and then $r\left(y\left(t^{+}\right)\right)$and $r\left(y\left(t^{-}\right)\right)$re at opposite sides of 1 .

From Proposition 8, $\left(y^{+}-y^{-}\right)-G\left(y^{+}, y^{-}\right)\left(y^{+}-y^{-}\right)=0$ and either $y^{+}-y^{-}>0$ or $\left(y^{+}-y^{-}\right)<0$. But $\left\langle\phi^{*}, y^{+}-y^{-}\right\rangle=\left\langle\phi^{*},\left(t^{+}-t^{-}\right) \phi\right\rangle>0$, so that $y^{+}-y^{-}>0$. By (r-Convs), $r\left(y\left(t^{+}\right)\right)>r\left(y\left(t^{-}\right)\right)$, so that $r\left(y\left(t^{+}\right)\right)>1>r\left(y\left(t^{-}\right)\right)$. From Theorem 3 (iii) and $F$ s not injective (item (ii)), $h$ is a (strictly) unimodal function. From Lemma $5, F$ folds downwards.

Proof of Theorem 3 (iv): Combine Propositions 10 and 13 (iii), as stated in the beginning of the section.

Corollary 3. The critical points of $F: Y \rightarrow Y$ are topological folds.

Proof: It suffices to show that the critical points of $F^{a}: W \oplus \mathbb{R} \rightarrow W \oplus \mathbb{R}$ are folds. Indeed, when restricted to each vertical line of the domain, $F^{a}$ is a proper, unimodal function folding downwards. The construction of local charts leading to the normal form of a (topological) fold is left to the reader.

\subsection{New folds from regularity}

Functions $\mathbf{F}=L-P: X \rightarrow Y$ between Sobolev spaces sometimes restrict to functions $\mathbf{G}: R \rightarrow Z$ between Hölder spaces. This is the case for the Dirichlet Laplacian $L=-\Delta_{D}$ acting on a bounded domain $\Omega \subset \mathbb{R}^{n}$ and a Nemitskii map $P(u)=f(u)$ for a smooth function $f: \mathbb{R} \rightarrow \mathbb{R}([2]$, [8]). Here, $n>1$, and $X=W^{2, n} \cap W_{0}^{1, n}, Y=L^{n}, R=C_{0}^{2, \alpha}, Z=C^{0, \alpha}$, where we drop the reference to the bounded set $\Omega \subset \mathbb{R}^{n}$. In both scenarios, $\mathbf{F}$ folds downwards.

Hölder spaces are not reflexive, but there Nemitskii operators have better differentiability properties. The non-normal cone of nonnegative continuous functions in $C^{0, \alpha}(\Omega)$ has a nonempty interior.

There is a feature in the example which was not explored in Theorem 3 (iv). As in Section 2.5, from $L: X \rightarrow Y$ we define $T=\iota \gamma(L+\gamma I)^{-1}$, and then systematically operate with the composition $y \mapsto P T(y)$ of functions from $Y$ to itself. Instead, we interpret $P T$ as the composition $\tilde{P}: X \rightarrow Y$ and $\tilde{T}: Y \rightarrow X$ : $\tilde{P}$ may have better smoothness properties than $P$.

The following amplification of Theorem 3 (iv) explores this feature. 
Theorem 6. Suppose $Y$ and $K$ as above, $\iota: X \rightarrow Y$ an inclusion. For some $\tilde{T} \in \mathcal{B}(Y, X)$, let $T=\iota \tilde{T} \in \mathcal{B}(Y)$ an r-special operator for which $r(T)=1, T \phi=$ $\phi>0$. Suppose $\tilde{P}: X \rightarrow Y$ satisfies the following properties, for some $\mathbf{b}>0$.

$(r-H s) \tilde{P}: X \rightarrow Y$ is a $C^{1}$ map such that $\tilde{J}(x)=D \tilde{P}(x) \in \mathcal{B}(X, Y)$ admits an extension $J(x) \in \mathscr{P}^{b}$.

(r-Convs) Let $y, z \in Y, y>z$. Then $J(\tilde{T} y)-J(\tilde{T} z) \in \mathcal{B}(Y)$ is strictly positive.

(r-Crit) $P T=\tilde{P} \tilde{T}: Y \rightarrow Y$ is $C^{1}$ and some $y \in Y$ is critical: $0 \in \sigma(D F(y))$.

Then $F: Y \rightarrow Y, y \mapsto y-P(T y)$ folds downwards.

The proof of Theorem 6 is identical to the proof of Theorem 3 (iv).

Theorem 7 provides a situation in which one fold gives rise to another. We present it in the geometric context of Theorem 3: say $F: Y \rightarrow Y$ and $G: Z \rightarrow Z$ admit a common expression - can they share geometric properties?

Theorem 7. Let $Y$ and the map $F=I-P T: Y \rightarrow Y$ be as in Theorem 6, so that $F$ folds downwards with respect to $\phi=\phi(T) \in Y$. Let $Z$ be a Banach space, with $v: Z \rightarrow Y$ be a dense inclusion and $G: Z \rightarrow Z$ be a $C^{1}$ restriction of $F$ such that $F \circ v=v \circ G$. Moreover, assume that (i) $T Y \subset Z$, (ii) $P: Z \rightarrow Z$, (iii) For $z \in Z$, the maps $D G(z): Z \rightarrow Z$ are Fredholm operators of index 0 .

Then $G$ folds downwards along $\phi$.

In the example above, $X=W^{2, n} \cap W_{0}^{1, n} \hookrightarrow C^{0, \alpha}=Z$, for $\alpha \in(0,1)$. For a Nemitskii map $P$ associated with a smooth function $f, \tilde{P}: X \rightarrow Z$ is $C^{1}$. The remaining hypotheses are standard.

Proof: By (i), $\phi \in Z$. From (ii), for $z \in Z$, the solutions $y$ of $F(y)=z \in Z$ are necessarily in $Z$. Indeed, write $F(y)=z$ as $y=P(T y)+z$. As $T: Y \rightarrow Z$, by (i), the right hand side is necessarily in $Z$ and $y \in Z$.

Moreover, $V=\langle\phi\rangle \subset Z \subset Y$ and $\phi^{*} \in Y^{*} \subset Z^{*}$. Decompose $Y=W_{Y} \oplus V$, for $W_{Y}=\operatorname{ker} \phi^{*}\left(\operatorname{as} \phi^{*} \in Y^{*}\right)$ and $Z=W_{Z} \oplus V$, for $W_{Z}=\operatorname{ker} \phi^{*}\left(\operatorname{as} \phi^{*} \in Z^{*}\right)$. The four projections are continuous and, for $z \in Z \subset Y$, both decompositions coincide: if $z=w_{Z}+t_{Z} \phi=w_{Y}+t_{Y} \phi$, then $w_{Z}=w_{Y}$ and $t_{Z}=t_{Y}$.

Vertical lines $L_{y}=\{y+s \phi, s \in \mathbb{R}\} \subset Y$ either lie in $Z$ or do not intercept it. Indeed, if $y \in Z$, then $y+s \phi \in Z$, by hypothesis (i). The same happens to fibers: if some point of the fiber $\Lambda_{y}=F^{-1}\left(L_{y}\right)$ lies in $Z$, then the whole fiber does. Indeed, if $F^{-1}\left(y+h_{0} \phi\right) \in Z$, we have $y \in Z$, from the implication of (ii) in the beginning of the proof, and then $F^{-1}(y+h \phi) \in Z$ for all $h \in \mathbb{R}$. Thus, for $y \in Z$, the fiber $\Lambda_{y}$ is the same for $F$ and $G$.

By hypothesis, $Y$ is foliated by its fibers $\Lambda_{y}$ and, from the previous fact, the same occurs to $Z$. Continuity is missing: we prove that fibers in $Z$ are curves.

From Proposition 4, the map $\Psi_{F}: W_{Y} \oplus \mathbb{R} \rightarrow W_{Y} \oplus \mathbb{R},(w, t) \mapsto\left(F^{t}(w), t\right)$ is a diffeomorphism. Its restriction $\Psi_{G}: W_{Z} \oplus \mathbb{R} \rightarrow W_{Y} \oplus \mathbb{R},(w, t) \mapsto\left(G^{t}(w), t\right) \in Z$ 
is clearly a $C^{1}$ map. It is also a bijection, since inverses of points $z \in Z$ under $F$ lie in $Z$. It is a global diffeomorphism: it suffices to show it is locally so. We proceed to apply the inverse function theorem.

Clearly, it is a matter of verifying the invertibility of $D G^{t}: W_{Z} \rightarrow W_{Z}$ for each $t$ fixed. Explicitly, $D G^{t}(w) v=\Pi(v-D P(T w+t T \phi) T v)$. Consider the inclusion $\iota: W_{Z} \rightarrow Z$, and write $D G^{t}(w)=\Pi \circ D G(w+t \phi) \circ \iota$, so that, by (iii), $D G^{t}(w)$ is a Fredholm operator of index 0. Thus, $D G^{t}(w)$ is not invertible if and only if it has a nontrivial kernel: let $\psi \in \operatorname{Ker} D G^{t}(w), \psi \neq 0$. As $D G^{t}(w)$ is a restriction of $D F^{t}(w)$, we must have $\psi \in \operatorname{Ker} D F^{t}(w)$, contradicting the fact that $\Psi_{F}$ is a diffeomorphism. Thus, $\Psi_{G}$ is a local, hence global diffeomorpshim.

The fibers of $G$ are those $F$, which folds downwards: $G$ does too.

\section{The effect of self-adjointness}

Let $H=L^{2}(M, d \nu)$ be a real Hilbert space with norm $|\cdot|$ for a $\sigma$-finite measure space $(M, \nu)$. Suppose $L: D \subset H \rightarrow H$ is a self-adjoint operator. We make no reference to the complexifications required for the underlying spectral theory.

The concepts related to positivity in both sections overlap, but we keep the more familiar terms in each context. A function is positive, $u \geq 0$ if $u(x) \geq 0$ a.e. and $u \neq 0$ and strictly positive, $u>0$ if $u(x)>0$ a.e.. The it positive cone $K \subset H$ is the set containing the positive functions and zero. A bounded operator $A: H \rightarrow H$ is positivity preserving if $A u \geq 0$ for all $u \geq 0$. It is positivity improving if for any $u \geq 0$, we have $A u>0$ and positively stable if $A u>0$ whenever $u>0$.

For appropriate self-adjoint operators $L: D \subset H \rightarrow H$ and maps $P: H \rightarrow H$, we consider counterparts to Theorem 3 for $F: D \rightarrow H, F(u)=L u-P(u)$.

\section{1 m-special operators and fine perturbations}

Let $L: D \subset H \rightarrow H$ be a self-adjoint operator. An eigenvalue $\lambda \in \sigma(L)$ of is basic if $\lambda \in \mathbb{R}$ is an isolated point of $\sigma(L)$ and the associated invariant eigenspace is spanned by a strictly positive eigenvector $\phi \in D, \phi>0$.

Set $\lambda_{1}=\min \sigma(L)$. A self-adjoint operator $L: D \rightarrow H$ is $m$-special if

$(\mathrm{m}-\mathrm{S}) \lambda_{1}$ is a basic eigenvalue; if $0 \in \sigma(L)$ is an eigenvalue, then $0=\lambda_{1}$.

(m-PI) $(L-\mu)^{-1}$ is positivity improving for all $\mu<\lambda_{1}$.

The operators $L$ or $(L-\mu)^{-1}$ are not required to be compact.

Set $\lambda_{2}=\inf \sigma(L) \backslash\left\{\lambda_{1}\right\}$. An operator $A \in \mathcal{B}(H)$ is a fine perturbation of $L$ if, for some $b, p \in \mathbb{R}$, it belongs to

$$
\mathscr{P}^{b, p}=\left\{A \in \mathcal{B}(H) \text { is symmetric, }\|A\| \leq b<\lambda_{2}\right. \text {, and }
$$




$$
A+p I \in \mathcal{B}(H) \text { is positive preserving. }\}
$$

We prove that, for a fine perturbation $A$ of the m-special operator $L$, the operator $L-A$ is m-special. We quote Theorem XIII.44 of [28] and the m-special case of a result by Faris [21] (also exercise 91, Chapter XIII of [28]).

Lemma 6. Let $L: D \rightarrow H$ be a self-adjoint operator such that $\lambda_{1}=\min \sigma(L)$ is an eigenvalue and $e^{-t L}$ is positivity preserving for $t>0$. Then $\lambda_{1}$ is a basic eigenvalue if and only if $L$ satisfies ( $m-P I)$, or equivalently,

(m-PI2) For all $t>0$, the operators $e^{-t L}: H \rightarrow H$ are positivity improving.

Lemma 7. Let $L: D \rightarrow H$ be a self-adjoint operator for which ( $m$-PI) holds and let $A: H \rightarrow H$ be a positivity preserving, bounded, symmetric operator. Then (m-PI) (hence (mPI2)) holds for $L-A: D \rightarrow H$.

Proposition 14. Let $L: D \rightarrow H$ be an m-special operator and let $A \in \mathscr{P}^{b, p}$ be a fine perturbation of $L$. Then $S=L-A: D \rightarrow H$ is $m$-special.

Proof: By the Kato-Rellich theorem, $S: D \rightarrow H$ is self-adjoint. Since min $\sigma(L)$ is basic, a standard argument shows that $\sigma_{m}=\inf \sigma(S)$ is necessarily a simple eigenvalue associated with a normal eigenvector $\psi_{m}$. We now prove that if 0 is an eigenvalue of $S$, and then then $0=\lambda_{1}$. Consider the quadratic form $Q(v)=$ $\langle S v, v\rangle$ for a normal $v \in D$. We must have $Q\left(\psi_{m}\right)=0$. If $\sigma_{m}<0$ there is a normal $\psi \in D$ for which $Q(\psi)<0$ and then $Q \leq 0$ in the plane $E$ spanned by $\psi_{m}$ and $\psi$. For a normal $v \in W_{D}=\phi_{1}^{\perp} \subset D,\langle L v, v\rangle \geq \lambda_{2}$ and $Q(v) \geq \lambda_{2}-\sup \sigma(A)>0$. But $E$ must intersect the (closed) codimension one subspace $W_{D}$, a contradiction. This settles (m-S) for $S$.

We now prove (m-PI2). For $t>0, e^{-t T}$ is positivity improving by (m-PI) for $L$. For some $p, p I+A$ is positive preserving. From Lemma $6, e^{-t S}=e^{-t p I} e^{-t(S-p I)}=$ $e^{-t p} e^{-t(L-(A+p I))}$ is positivity improving.

\subsection{Counterparts to Theorem 3}

Let $\phi_{1}>0$ be the normal eigenvector associated with $\lambda_{1}=\lambda_{1}(L)$. Set $V=$ $\left\{t \phi_{1}, t \in \mathbb{R}\right\}$ and consider the orthogonal decompositions $H=W \oplus V, D=$ $W_{D} \oplus V$, for $W_{D}=(W \cap D)$. Let $\Pi$ be the orthogonal projection $\Pi: H \rightarrow W$.

As in the previous section, a linearization of $F=L-P$ is a bounded, symmetric operator $G(u, v) \in \mathcal{B}(H)$ such that, for $u, v \in D \subset H, F(v)-F(u)=$ $(L-G(v, u))(v-u)$. Again, if $P: H \rightarrow H$ is a $C^{1}$ map, linearizations are obtained from Jacobians $J(u)=D P(u): H \rightarrow H \in \mathcal{B}(H)$,

$$
F(v)-F(u)=\left(L-\int_{0}^{1} D P(u+t(v-u)) d t\right)(v-u):=(L-G(v, u))(v-u) .
$$

Consider the following properties for the Lipschitz map $P: H \rightarrow H$. 
(m-H) There are $b, p \in \mathbb{R}$ such that, for $u, v \in D, \Pi G(u, v) \in \mathscr{P}^{b, p}$.

(m-Hs) There are $b, p \in \mathbb{R}$ such that, for $u \in D, \Pi D P(u) \in \mathscr{P}^{b, p}$.

(m-Conv) If $u<v<w$, then $\langle w-v, F(v)-F(u)\rangle\langle\langle v-u, F(w)-F(v)\rangle$ or, equivalently, $\langle v-u, P(w)\rangle+\langle w-v, P(u)\rangle+\langle u-w, P(v)\rangle>0$.

(m-Convs) $P: H \rightarrow H$ is a $C^{1}$ map, $u, v \in H, v>u$. Then $D P(v)-D P(u) \in \mathcal{B}(H)$ is positivity preserving.

(m-Crit) $P: H \rightarrow H$ is $C^{1}$ and some $u \in H$ is critical: $0 \in \sigma(D F(y))$.

Again,(m-Hs) and (m-Convs) imply (m-H) and (m-Conv) respectively.

Theorem 8. Suppose $H$ as above, $L: D \subset H \rightarrow H$ an $m$-special operator, and $P: H \rightarrow H$ a Lipschitz (or $C^{1}$ ) map admitting linearizations $G(u, v)$. Define the function $F: D \rightarrow H, u \mapsto L u-P(u)$.

(i) If $\left[-\sup _{u, v}\|G(u, v)\|, \sup _{u, v}\|G(u, v)\|\right] \cap \sigma(L)=\emptyset$, then $F$ is a homeomorphism.

(ii) Suppose that (m-H), (m-Conv) hold. Then $F$ is a simple map.

(iii) If (m-H), (m-Conv) hold and $F: H \rightarrow H$ is proper, then it is a homeomorphism or folds vertically.

(iv) Suppose that (m-Hs), (m-Convs) and (m-Crit) hold. Then F folds downwards.

There are no restrictions on $b$ besides $b<\lambda_{2}$ : Theorem 3 is more demanding.

The proof follows closely the arguments of the previous section. For $t \in \mathbb{R}$, set $P^{t}: W \rightarrow W, P^{t}(w)=\Pi P\left(w+t \phi_{1}\right)$ and $F^{t}: W_{D} \rightarrow W, F^{t}(w)=\Pi F\left(w+t \phi_{1}\right)$.

Proposition 15. Suppose that $(m-H)$ holds. Then, for each $t \in \mathbb{R}$, the maps $F^{t}: W_{H} \rightarrow W$ and $\Psi: W \oplus \mathbb{R} \rightarrow W \oplus \mathbb{R}, \Psi(w, t)=\left(F^{t}(w), t\right)$ are bilipschitz homeomorphisms. Thus

$$
F^{a}(z, t)=F \circ \Psi^{-1}(z, t)=\left(z, h^{a}(z, t)\right),
$$

for a Lipschitz height function $h^{a}: Y=W \oplus V \rightarrow \mathbb{R}$. The map $F$ admits fibers $\Lambda_{z}=\left\{u(t)=w(t)+t \phi_{1}, t \in \mathbb{R}\right\}=F^{-1}\left(L_{z}\right)$ and $F^{a}\left(L_{z}\right)=\Lambda_{z}$.

The argument is standard ([8], [11], [13], [27], [29], [30], [31], [39]). The positivity of the eigenfunction associated with $\lambda_{1}$ is not required, nor the existence of positive maps $A+p I$ in the definition of $\mathscr{P}^{b, p}$.

Proof: Since $L$ is m-special and $W_{D}=V^{\perp}$, the restriction $L_{W}: W_{D} \rightarrow W$ is invertible: $\sigma\left(L_{W}\right)=\sigma(L) \backslash\left\{\lambda_{1}\right\}$. To show the invertibility of $F^{t}: W \rightarrow W$ for each $t \in \mathbb{R}$, we solve $F^{t}(w)=\Pi L\left(w+t \phi_{1}\right)-\Pi P\left(w+t \phi_{1}\right)=z$, for $z \in W$, or

$$
y=P^{t}\left(L_{W}^{-1} y\right)+z \quad \text { for } \quad y=L_{W} w \in W .
$$


From $(\mathrm{m}-\mathrm{H})$, the map $K^{t}(y)=P^{t}\left(L_{W}^{-1} y\right)$ is a contraction, with constant $c=b / \lambda_{2}$ independent of $t$. Indeed,

$$
\begin{aligned}
& \left|K^{t}\left(w_{1}\right)-K^{t}\left(w_{2}\right)\right|=\left|\Pi\left(P\left(L_{W}^{-1}\left(w_{1}\right)+t \phi_{1}\right)-P\left(L_{W}^{-1}\left(w_{2}\right)+t \phi_{1}\right)\right)\right| \\
& =\left|\Pi G\left(L_{W}^{-1} w_{1}+t \phi_{1}, L_{W}^{-1} w_{2}+t \phi_{1}\right) L_{W}^{-1}\left(w_{1}-w_{2}\right)\right| \leq \frac{b}{\lambda_{2}}\left|w_{1}-w_{2}\right|,
\end{aligned}
$$

as $\left\|L_{W}^{-1}\right\|=1 / \lambda_{2}$ and, by $(\mathrm{m}-\mathrm{H}),\|\Pi G\| \leq b<\lambda_{2}$ : $K^{t}$ is a contraction. Set $c=b / \lambda_{2}$.

Hence the maps $I-K^{t}: W \rightarrow W$ are (uniform) Lipschitz bijections. The inverses $\left(I-K^{t}\right)^{-1}: W \rightarrow W$ are also uniformly Lipschitz with constant $1 /(1-c)$ : for $z_{i}=\left(I-K^{t}\right)^{-1}\left(y_{i}\right), i=1,2$,

$$
\left|z_{1}-z_{2}\right| \leq\left|y_{1}-y_{2}\right|+\left|K^{t}\left(z_{1}\right)-K^{t}\left(z_{2}\right)\right| \leq\left|y_{1}-y_{2}\right|+c\left|z_{1}-z_{2}\right| .
$$

Thus $F^{t}=\left(I-K^{t}\right) \circ L_{W}$ are also uniformly bilipschitz homeomorphisms. The map $\Psi=\left(F^{t}, \mathrm{Id}\right): D \rightarrow H$ is a homeomorphism because of the continuous dependence of the fixed point with respect to $t$.

\section{Remarks}

1. To use Proposition 15 with the hypotheses of Theorem 1, translate $F=\mathbf{F}-\gamma I$, $P=\mathbf{P}-\gamma I$ with $\gamma=(a+b) / 2$.

2. In Theorem $1, P(u)=f(u)$ and the values of $f^{\prime}$ are close to $\lambda_{1}: P(u)$ is roughly a multiplication by $\lambda_{1}$ (plus a constant). For a general $P$, once the projected maps $\Pi P^{t}$ are appropriately bounded, adapted coordinates apply, the behavior of the map $F$ along the vertical axis may be different.

3. Under the hypotheses of Theorem 8 (i), the estimates in the proof apply to $F: D \rightarrow H$ itself: $F$ is a bilipschitz homeomorphism. This is the usual DolphHammerstein theorem ([18], [23]).

From Proposition 15, vertical lines $L_{z}=\left\{z+h \phi_{1}, h \in \mathbb{R}\right\} \subset H$, when inverted by $F$, give rise to fibers $\Lambda_{z}=\left\{u(t)=w(t)+t \phi_{1}, w(t) \in W_{D}, t \in \mathbb{R}\right\}$.

Without loss, after a translation in the range $H$, we assume $P(0)=F(0)=0$. Indeed, hypotheses $(\mathrm{m}-\mathrm{H})$ and $(\mathrm{m}-\mathrm{Conv})$ are invariant under this operation.

Corollary 4. Let $\Lambda_{z}$ be a fiber. For some $C \in \mathbb{R},|w(t)| \leq C\left(|z|+\left|\Pi P\left(t \phi_{1}\right)\right|\right)$.

Proof: Since $F^{t}: W_{D} \rightarrow W$ are bilipschitz maps and $F(u(t))=z+h(t) \phi_{1}$,

$$
\begin{gathered}
|w(t)-0| \leq C\left|F^{t}(w(t))-F^{t}(0)\right| \\
=C\left|\Pi F\left(w(t)+t \phi_{1}\right)-\Pi F\left(t \phi_{1}\right)\right|=C\left|z-\Pi P\left(t \phi_{1}\right)\right|
\end{gathered}
$$

and the result follows. 
Adapted coordinates provide examples. For $-\Delta: H^{2}(\Omega) \cap H_{0}^{1}(\Omega) \rightarrow H^{0}(\Omega)$, with smallest eigenvalue $\lambda_{1}$ and ground state $\phi_{1}$, consider, for $t=\left\langle\phi_{1}, u\right\rangle$,

$$
F(u)=L u-P(u)=\left(-\Delta-\lambda_{1}\right) u+(I-\Pi)(\sin t) u,
$$

The change of variables $z=\left(-\Delta-\lambda_{1}\right) w$ yields $F^{a}(z, t)=(z, t \sin t)$, an example of a function $F$ with points with infinitely many preimages.

Proposition 16. If $F(u)=F(v)$ and $P$ satisfies (m-Conv), then either $u>v$ or $u=v$ or $u<v$. If (m-Conv) also holds, $F(u)=g$ has at most two solutions.

Proof: An integral of fine perturbations as in Equation 2 for $G(u, v)$, is also fine. By Proposition 14, $L-G(u, v)$ is m-special. If $F(u)=F(v)$, then $\pm(u-v) \in$ $\operatorname{Ker}(L-G(u, v))$ is a positive eigenfunction or 0 , by $(\mathrm{m}-\mathrm{S})$ : trichotomy holds.

Suppose $F(u)=F(v)=F(w)=g$. By the previous paragraph, we may assume $u<v<w$, and then the first form of (m-Conv) is clearly violated.

Proof of Theorem 8: Item (i) is a remark after Proposition 15. Proposition 15 showed that no additional restrictions on $b>0$ are necessary. Proposition 16, in turn, employed (m-Conv) exactly as Proposition 6 employed (r-Conv). The proof now follows the arguments in the previous section.

\subsection{Asymptotics of heights: properness}

Informally, if (m-H) holds, the properness of $F$ depends on its behavior along the vertical axis $V=\left\langle\phi_{1}\right\rangle$. Assume $P: H \rightarrow H$ Lipschitz and recall $F(0)=0$. Set

$$
F\left(t \phi_{1}\right)=z_{v}(t)+h_{v}(t) \phi_{1}=\Pi P\left(t \phi_{1}\right)+\left\langle\phi_{1}, F\left(t \phi_{1}\right)\right\rangle \phi_{1}, \text { for } z_{v} \in W \text {. }
$$

Proposition 17. Suppose the function $F: D \rightarrow H$ satisfies $(m-H)$ and

(m-hor) $\lim _{|t| \rightarrow \infty}\left|z_{v}(t)\right|_{H} / t=0$.

(m-ver) For some $c>0$ and large $|t|,\left|h_{v}(t)\right| \geq c|t|$.

Then $F$ is proper.

From Corollary 4, $|w(t)| \leq C\left(|z|+\left|z_{v}(t)\right|\right)$. Hypothesis (m-hor) implies that fibers $w(t)+t \phi_{1}$ are asymptotically vertical: indeed, $w(t) / t \rightarrow 0$ for $|t| \rightarrow \infty$. Hypothesis (m-ver) imposes a rate of growth for the height $h_{v}(t)$.

Proof: For $z \in W$, consider the fiber $\Lambda_{z}=\left\{u(t)=w(t)+t \phi_{1}\right\}$, and its image $F(u(t))=z+h_{z}(t) \phi_{1}$. As $L W_{D} \subset W$,

$$
\begin{gathered}
h_{z}(t)=\left\langle\phi_{1}, L\left(w(t)+t \phi_{1}\right)-P\left(w(t)+t \phi_{1}\right)\right\rangle=\lambda_{1} t-\left\langle\phi_{1}, P\left(w(t)+t \phi_{1}\right)\right\rangle \\
=\lambda_{1} t-\left\langle\phi_{1},\left(P\left(w(t)+t \phi_{1}\right)-P\left(t \phi_{1}\right)\right)\right\rangle-\left\langle\phi_{1}, P\left(t \phi_{1}\right)\right\rangle
\end{gathered}
$$


Since $P: H \rightarrow H$ is Lipschitz, from Corollary 4,

$$
\left|P\left(w(t)+t \phi_{1}\right)-P\left(t \phi_{1}\right)\right| / t \leq C_{1}|w(t) / t| \leq C_{2}\left|\Pi P\left(t \phi_{1}\right) / t\right|+o(1),
$$

for constants $C_{i}$ locally uniformly in $z$. From (m-hor), for large $|t|$,

$$
h_{z}(t) / t=\lambda_{1}-\left\langle\phi_{1}, P\left(t \phi_{1}\right)\right\rangle / t+o(1)=h_{v}(t) / t+o(1) .
$$

Thus $h_{v}$ and $h_{z}$ have the same rate of growth, given by (m-ver).

We prove the equivalent properness of $F^{a}=F \circ \Psi^{-1}$. The estimates for $h_{z}$ translate to $h_{z}^{a}=h_{z} \circ \Psi^{-1}$, as $\Psi: W_{D} \oplus \mathbb{R} \rightarrow W \oplus \mathbb{R}$ in Proposition 15 leaves the vertical component unaltered. For a convergent sequence in the image of $F^{a}$, $\left(z_{n}, s_{n}\right)=\left(z_{n}, h_{z}^{a}\left(z_{n}, t_{n}\right)\right) \rightarrow\left(z_{\infty}, s_{\infty}\right)$. The estimates for $h^{a}$, being locally uniform in $z$, imply the boundedness of $\left\{t_{n}\right\}: F^{a}$ is proper.

Under the hypotheses of Theorem 8 , if the asymptotic signs of $h_{v}(t)$ are different then $F$ is a homeomorphism. If they are equal, $F$ is a global fold.

\subsubsection{Nemitskii maps yield properness and folds}

This natural extension of Theorem 1 is a counterpart of Theorem 5 .

Theorem 9. Suppose $H$ as above, $L: D \subset H \rightarrow H$ an $m$-special operator such that $\phi_{1} \in L^{1}$. Let $P: H \rightarrow H$ be a Nemitskii map $P(u)=f(u)$ for a strictly convex function $f: \mathbb{R} \rightarrow \mathbb{R}$ whose Newton quotient satisfies

$$
-\infty<a=\inf _{r \neq s} q(r, s)<\lambda_{1}<b=\sup _{r \neq s} q(r, s)<\lambda_{2} .
$$

Then the function $F: D \rightarrow H, u \mapsto L u-P(u)$ folds downwards

The functions in $H$ do not have to be defined on bounded domains.

We obtain the properness of $F$ from Proposition 17.

Lemma 8. (i) $P(u)=f(u)-(b+a) u / 2$ satisfies $(m-H)$ and ( $m$-Conv).

(ii) (m-hor) holds.

(iii) (m-ver) also holds: $\lim _{t \rightarrow-\infty} h_{v}(t) / t=\lambda_{1}-a>0, \lim _{t \rightarrow \infty} h_{v}(t) / t=\lambda_{1}-b<0$.

Proof: (i) The verification of properties $(\mathrm{m}-\mathrm{H})$ and (m-Conv) is by now familiar.

(ii) For $v \in W_{D}$ of norm one and compact support (and then $\Pi v=v$ ),

$$
\left\|\Pi P\left(t \phi_{1}\right) / t\right\|_{H}=\sup _{|v|=1}\left|\left\langle v, \Pi P\left(t \phi_{1}\right) / t\right\rangle\right|=\sup _{|v|=1}\left|\left\langle v, f\left(t \phi_{1}\right) / t\right\rangle\right| .
$$

We use the dominated convergence theorem. We consider $t \rightarrow \infty$, the other case is similar. By the convexity of $f$, for $x>0, f(t x) / t \rightarrow b x$ if $t \rightarrow \infty$, and the integrand $\left|v f\left(t \phi_{1}\right) / t\right|$ is pointwise bounded by an $L^{1}$ function. Taking limits,

$$
\left\|\lim _{t \rightarrow \infty} \Pi P\left(t \phi_{1}\right) / t\right\|_{H}=\sup _{|v|=1}\left|\int_{\Omega} \lim _{t \rightarrow \infty} v f\left(t \phi_{1}\right) / t\right|=\sup _{|v|=1}\left|b \int_{\Omega} v \phi_{1}\right|=0
$$

since $v \in W_{D}=V^{\perp}$. Statement (iii) also follows from dominated convergence. 
Proof of Theorem 9: Combine Theorem 8 (iii) with Lemma 8 (iii).

A Lipschitz function $f$ induces a Lipschitz Nemitskii map $P: L^{2}(\Omega) \rightarrow L^{2}(\Omega)$ on unbounded sets $\Omega \subset \mathbb{R}^{n}$ if $f(0)=0$. As an example of application of Theorem 9, consider the m-special (see Proposition 3.4.1) Hamiltonian for the hydrogen atom in $\mathbb{R}^{3}, L u=-\Delta u-u / r$, with basic eigenvalue $\lambda_{1}(L)$ associated with a normal, positive ground state $\phi_{1} \in L^{1} \cap L^{2}$.

\subsection{Some examples}

Folds related to self-adjoint elliptic operators different from the Dirichlet Laplacian are known $([4])$ : we present some more. The larger class of $m$-special operators $L: D \rightarrow H$ includes the finite dimensional case $D=H=\mathbb{R}^{n}$ when $\nu$ is a finite collection of deltas and the operators described in Proposition 18.

\subsection{1 m-special operators $L$}

The identification of operators $L$ generating positivity preserving semigroups (hypothesis (m-PI2)) is by itself a field of mathematics. Arguments in the spirit of Bochner's theorem on distributions of positive type and the Levy-Khintchine formula (Appendix 2, [28], vol. IV) lead to a wealth of examples.

Proposition 18. The following operators are m-special: I. $-\Delta$ for Dirichlet, Neumann, periodic or mixed boundary conditions on bounded smooth domains; II. The hydrogen atom in $\mathbb{R}^{3}, L u=-\Delta u-u / r$; III. The quantum oscillator $L u(x)=-u^{\prime \prime}(x)+x^{2} u(x) ; I V$. Fractional powers $L^{s}, s \in(0,1)$ of positive $m$ special operators.

Proof: Hypothesis (m-S) is familiar for all examples, we check (m-PI). For (I), see Sections 8.1 and 8.2 of [3]. For (II), take $L_{0}=-\Delta$ with $D=H^{2}\left(\mathbb{R}^{3}\right)$ and define $L=L_{0}+V$ for the potential $V=-1 / r$. We prove (m-PI) for $L$ using Theorem XIII.45, vol. IV of [28]. Define the bounded truncations $V_{n}$ which coincide with $V$ for $|x|>1 / n$ and are zero otherwise. Set $q_{n}=V-V_{n}$. Both $L_{0}$ and $L$ are bounded from below. Comparing quadratic forms,

$$
L \leq L_{0}+V_{n} \text { and } L_{0} \leq L-V_{n},
$$

so that $L_{0}+V_{n}$ and $L-V_{n}$ are uniformly bounded from below. We are left with showing that $L_{0}+V_{n} \rightarrow L$ and $L-V_{n} \rightarrow L_{0}$ in the strong resolvent sense. By Theorem VIII.25, vol. I of [28] it suffices to show that, for a given $u \in H^{2}$,

$$
q_{n} u \rightarrow 0 \quad \text { in } L^{2}\left(\mathbb{R}^{3}\right), \quad \text { i.e. } \lim _{\epsilon \rightarrow 0} \int_{|x| \leq \epsilon} \frac{u^{2}(x)}{|x|^{2}} d x=0
$$

which is true, since $H^{2}\left(\mathbb{R}^{3}\right)$ consists of bounded, continuous functions. The proof of (III) is similar. For (IV), use the arguments with Laplace transforms in Section IX.11 of [42] (see also [38]). 
An m-special operator $L: D \rightarrow H$ with $\lambda_{1}>0$ yields m-special operators $L \otimes L, L \wedge L, L \otimes I+I \otimes L$ in appropriate domains.

There are natural m-special operators associated to systems. For a finite measure space $(M, d \nu)$, let $(\tilde{M}, d \nu)$ be the disjoint union of $k$ copies of $(M, d \nu)$. Clearly, for $H=L^{2}(M, d \mu)$, we have $\tilde{H}=L^{2}(\tilde{M}, d \nu) \simeq H^{k}$. Inequalities between vectors must hold componentwise. We consider a simple example.

Let $L: D \rightarrow H$ be m-special, $\lambda_{1}(L)=\min \sigma(L)$ associated with $\phi_{1}(L)>0$, $\lambda_{2}(L)=\inf \sigma(L) \backslash \lambda_{1}(L)$. Then, for $\alpha>0$ with $-\lambda_{1}(L)<\alpha<\lambda_{2}(L)$,

$$
\tilde{L}: \tilde{D}=D \oplus D \mapsto \tilde{H}=H \oplus H,\left(u_{1}, u_{2}\right) \mapsto\left(L u_{1}-\alpha u_{2}, L u_{2}-\alpha u_{1}\right)
$$

is m-special. We have $\tilde{\lambda}_{1}=\min \sigma(\tilde{L})=\lambda_{1}(L)-\alpha$ and ground state $\sqrt{2} \phi_{1}=$ $\left(\phi_{1}(L), \phi_{1}(L)\right)$. Also, $\tilde{\lambda}_{2}=\inf \left(\sigma(\tilde{L}) \backslash\left\{\tilde{\lambda}_{1}\right\}\right)=\min \left\{\lambda_{1}(L)+\alpha, \lambda_{2}(L)-\alpha\right\}>0$, from which (m-S) follows; $\alpha>0$ in turn implies (m-PI).

\subsubsection{Compatible maps $P$}

From Proposition 14, we must find maps $P(u)$ admitting linearizations which are fine perturbations of an m-special operator $L$. We present two examples.

Consider maps $P(u)$ which are gradients of $\Psi: D \rightarrow \mathbb{R}$, so that the Jacobian $D P$ is a Hessian, hence symmetric with appropriate smoothness. Let $\Omega \subset \mathbb{R}^{n}$ bounded, $H=L^{2}(\Omega, d x), B, A \in \mathcal{B}(H)$ and $\mathbf{f}$ a primitive of $f, \mathbf{f}^{\prime}=f$, set

$$
\Psi: H \rightarrow \mathbb{R}, \quad u \mapsto \int_{\Omega} B \mathbf{f}(A u) d x
$$

for which, at least formally (here, $1 \in H$ is the constant function),

$$
P(u)=\nabla \Psi(u)=A^{T} f(A u)\left(B^{T} 1\right) .
$$

The usual Nemitskii map is the case $B=A=I$.

Proposition 19. Suppose $A \in \mathcal{B}(H)$ is positively stable, $g=B^{T} 1>0, f$ as in Theorem 9, $P(u)=A^{T} g f(A u)$. Then, if $\|A\|^{2}\left\|g f^{\prime}\right\|_{\infty} \leq b<\lambda_{2},(m-H)$ and (m-Conv) hold and thus $F=L-P$ is a simple map.

As $f$ is Lipschitz, $f^{\prime}$ exists a.e. A finer result may be obtained assuming smoothness, as in Proposition 10, but we give no details.

Proof: Take for linearizations the maps $G(u, v)=A^{T} M_{g q(u, v)} A$, where, as usual, $M_{g q(u, v)} \in \mathcal{B}(H)$ is multiplication by $g q(u, v) \in L^{\infty}(\Omega)$ and $q$ is the usual Newton quotient associated with $f$. The hypotheses immediately yield $(\mathrm{m}-\mathrm{H})$.

We consider (m-Conv). From the convexity of $f$, for $u<v<w$,

$$
(w-v)(f(v)-f(u))<(v-u)(f(w)-f(v)) .
$$


As $A$ is positively stable, $A u<A v<A w$ and

$$
(A w-A v) g(f(A v)-f(A u))<(A v-A u) g(f(A w)-f(A v))
$$

pointwise (for all $x \in \Omega$ ). Integrate over $\Omega$,

$$
\left\langle w-v, A^{T} g(f(A v)-f(A u))\right\rangle<\left\langle v-u, A^{T} g(f(A w)-f(A v))\right\rangle,
$$

so that $\langle w-v, P(v)-P(u)\rangle<\langle v-u, P(w)-P(v)\rangle$.

We consider invariant maps. For an orthogonal projection $\pi: H \rightarrow H$, split

$$
H=H_{k} \oplus H, \quad \text { for } H_{k}=\operatorname{Ker} \pi, H=\operatorname{Ran} \pi, \quad u=u_{k}+u .
$$

Suppose $L: D \subset H \rightarrow H$ is an invertible operator commuting with $\pi$ and $P: H \rightarrow H$ is a map keeping $H$ invariant (as a subspace) for which $P(0)=0$. There are two maps to consider: $F: D \rightarrow H, F(u)=L u-\pi \circ P \circ \pi$ and its restriction $F_{\pi}: D \cap H \rightarrow H$, which is necessarily of the form $F_{\pi}(u)=L u-P(u)$.

If the restriction $L_{\pi}: D \cap H \rightarrow H$ is m-special and $P_{\pi}: H \rightarrow H$ is $L$ compatible with $L$, Theorem 8 applies to $F$. We now consider $F$ : in order to solve $F(u)=g$, decompose the equation as

$$
L u-P(u)=g, \quad L u_{k}=g_{k}
$$

and the invertibility of $L$ implies that $F=F \oplus L: H \oplus H_{k} \rightarrow H \oplus H_{k}$, so that the implications of Theorem 8 and Proposition 17 are common to $F$ and $F_{\pi}$.

As a simple example, let $\pi$ be the radial projection on functions defined in a ball, $L=-\Delta$ with Dirichlet conditions and $P$ a Nemitskii map as in Theorem 1 .

\section{Appendix: Eigenvectors on cones, after Marek}

We will use a standard version of the Krein-Rutman theorem ([17]).

Theorem (Krein-Rutman). Let $K$ be a reproducing cone of a Banach space $Y$, $S$ a compact operator for which $S K \subset K$ and $r(S)>0$. Then $\lambda(S)=r(S)$ is an eigenvalue associated with an eigenvector $\phi \in K$.

Theorem 10 below implies Theorem 4 in Section 2.1. Theorem 10 is a subset of the statements in Theorems 2.1, 2.2 and 2.3 in [26], inspired by ideas in [24], [10], [32], [33] and [34]. Nonsupporting operators and $B$-cones will not be used in this text, and are defined in [26], [34].

Theorem 10. [Bonsall-Marek-Sawashima-Schaefer] Let $Y$ be a real Banach space and $K \subset Y$ be a normal $B$-cone of $Y$. Let $S \in \mathcal{B}(Y)$ be a positive, nonsupporting operator and suppose that the spectral radius $r(S)$ is a pole of the resolvent map $\lambda \mapsto R(\lambda, S)=(\lambda I-S)^{-1}$. Then the following properties hold. 
(i) There exists an eigenvector $\phi \in K$ associated with $r(S), \phi>0$.

(ii) The spectral radius $r(S)$ is a simple pole of $R(\lambda, S)$.

(iii) Every eigenvector of $S$ in $K$ is a multiple of $\phi$.

(iv) For an eigenvalue $\lambda \in \sigma(S)$ different from $r(S),|\lambda|<r(S)$.

(v) There is an eigenvector $\phi^{*} \in K^{*}$ of $S^{*}$ associated with $r(S), \phi^{*}>0$.

(vi) If $\mu>r(S)$, then $(\mu I-S)^{-1} \in \mathcal{B}(Y)$ and $(\mu I-S)^{-1} K \subset K$.

We prove Theorem 4 from this theorem. In Section 2.1, $Y$ is a real Banach space, $K \subset Y$ is a normal, reproducing cone. A reproducing cone is unflattened (from the Krein-Smulian theorem, [40]) and unflattened cones are B-cones ([34]). Also, $S \in \mathcal{B}(Y)$ is compact and ergodic with $r(S)>0$. Ergodicity implies that $S$ is positive, nonsupporting. From the Krein-Rutman theorem, $r(S)>0$ and the spectral theory of compact operators implies that $r(S)$ is a pole of the resolvent map. Hence, the hypotheses of Theorem 4 imply those of Theorem 10.

We show that $r$ is a basic eigenvalue of $S$. From (i) and (v), $S$ and $S^{*}$ have eigenvectors $\phi>0$ and $\phi^{*}>0$ associated with $r(S)$ : this is property (b-1). By the compactness of $S$ and $S^{*}$, the invariant subspaces $V_{r}$ and $V^{*}$ associated to $r(S)$ are finite dimensional and it is easy to see that $\operatorname{dim} V=\operatorname{dim} V^{*}$. The Jordan theorem for matrices applied to the restriction of $S$ in $V$ implies that the resolvent map $\lambda \rightarrow(S-\lambda I)^{-1}$ has a pole at $r(S)$ of multiplicity given by the dimension of $V$. By (ii), then, $V$ is one dimensional. This proves properties (b-2) and (b-3): $r(S)$ is a basic eigenvalue of $S$. The remaining claims of Theorem 4 are explicit consequences of Theorem 10.

\section{References}

[1] A. Ambrosetti, Osservazioni sui teoremi di inversione globale, Rend. Lincei Mat. Appl. 22 (2011) 3-15.

[2] A. Ambrosetti and G. Prodi, On the inversion of some differentiable mappings with singularities between Banach spaces, Ann. Mat. Pura Appl. 93 (1972) 231-246.

[3] W. Arendt, Semigroups and Evolution Equations: Functional Calculus, Regularity and Kernel Estimates, Handbook Differential Equations, Eds. C.M. Dafermos and E. Feireisl, vol. I (2004), Elsevier, Amsterdam, 1-86.

[4] H. Berestycki, Le nombre des solutions de certain problèmes sémi-linéaires elliptiques, J. Funct. Anal. 40 (1981) 1-29. 
[5] H. Berestycki, L. Nirenberg and S.R.S. Varadhan, The principal eigenvalue and maximum principle for second-order elliptic operators in general domains, Comm. Pure Appl. Math. 47 (1994) 47-92.

[6] M.S. Berger, Nonlinearity and Functional Analysis, Lectures on Nonlinear Problems in Mathematical Analysis, Academic Press (1997).

[7] M.S. Berger and P.T. Church, Complete integrability and perturbation of a nonlinear Dirichlet problem. I., Indiana Univ. Math. J. 28 (1979) 935-952.

[8] M.S. Berger and E. Podolak, On the solutions of a nonlinear Dirichlet problem, Indiana Univ. Math. J. 24 (1974) 837-846.

[9] I. Birindelli, Hopf's lemma and Anti-maximum Principle in General Domains, J. Diff. Eqs. 119 (1995) 450-472.

[10] F. F. Bonsall, Endomorphisms ofpartially ordered vector spaces, J. London Math. Soc. 30 (1955) 133-144.

[11] H. Brezis and L. Nirenberg, Characterizations of the ranges of some nonlinear operators and applications to boundary value problems, Ann. Scuola Normale Sup. Pisa, Classe di Scienze 4a serie, 5, 2 (1978), 225-326.

[12] J.T. Cal Neto and C. Tomei, Numerical analysis of semilinear elliptic equations with finite spectral interaction, J.Math.Anal.Appl. 395 (2012) 63-77.

[13] M. Calanchi, C. Tomei and A. Zaccur, Fibers and global geometry of functions, PNLDE 86, Springer, Cham (2015) 55-75.

[14] M. Calanchi, C. Tomei and A. Zaccur, Cusps and a converse to the Ambrosetti-Prodi theorem, Ann. Sc. Norm. Sup. Pisa (5) XVIII (2018) 483-507.

[15] P.T. Church and J.G. Timourian, Global fold maps in differential and integral equations, Nonlinear Anal. 18 (1992) 743-758.

[16] P.T. Church and J.G. Timourian, Global Structure for Nonlinear Operators in Differential and Integral Equations I. Folds, Topological Nonlinear Analysis, II, PNLDE 27, Birkhäuser, Boston, MA, (1997) 109-160.

[17] K. Deimling, Nonlinear Functional Analysis, Dover, New York (2010).

[18] C.L. Dolph, Nonlinear integral equations of the Hammerstein type, Trans. Amer. Math. Soc. 66 (1949) 289-307.

[19] N. Dunford and J. Schwartz, Linear Operators I, Interscience, New York (1957). 
[20] N. Eldredge, Compactness of unit ball in WOT of $\mathcal{B}(X)$, available at https://math.stackexchange.com/questions/1187844/

[21] W. Faris, Invariant cones and uniqueness of the ground state for Fermion systems, J. Math. Phys 13 (1972) 1285-1290.

[22] D. Guo, Y. Je Cho, J. Zhu, Partial ordering methods in nonlinear problems Nova Science Publishers, Inc., Hauppauge, NY (2004).

[23] A. Hammerstein, Nichtlineare Integralgleichungen nebst Anwendungen, Acta Math. 54 (1929) 117-176.

[24] S. Karlin, Positive Operators, J. Math. Mech. 8 (1959) 907-937.

[25] A. Manes and A.M. Micheletti, Un'estensione della teoria variazionale classica degli autovalori per operatori ellittici del secondo ordine, Boll. Unione Mat. Ital. 7 (1973) 285-301.

[26] I. Marek, Frobenius theory of positive operators: comparison theorems and applications, SIAM J. Appl. Math. 19 (1970) 607-628.

[27] I. Malta, N. Saldanha and C. Tomei, Morin singularities and global geometry in a class of ordinary differential operators, Topol. Meth. Nonlin. Anal. 10 (1997) 137-169.

[28] M. Reed and B. Simon, Methods of Modern Mathematical Physics, vols. I-IV, Academic Press, New York (1975-78).

[29] B. Ruf, Singularity theory and the geometry of a nonlinear elliptic equation, Ann. Scuola Norm. Sup. Pisa C1. Sc., (2), 17 (1990) 1-33.

[30] B. Ruf, Singularity theory and bifurcation phenomena in differential equations, Topological Nonlinear Analysis, II, Progr. Nonlinear Differential Equations Appl., 27, Birkhäuser, Boston, MA, (1997) 315-395.

[31] B. Ruf, Singularity theory and the bifurcation structure of a nonlinear elliptic equation Lecture Notes in Pure and Appl. Math. 127, (1989) 435-443.

[32] I. Sawashima, On spectral properties of some positive operators, Natur. Sci. Rep. Ochanomizu Univ., 151 (1964) 55-64.

[33] H.H. Schaefer, On the singularities of an analytic function with values in a Banach space, Arch. Math., 11 (1960) 40-43.

[34] H.H. Schaefer, Some spectral properties of positive linear operators, Pacific Journal of Mathematics, Vol. 10, No. 3 (1960), 1009-1019. 
[35] J. Schauder, Invarianz des Gebietes in Funktionalräumen, Studia Math., 1 (1929)123-139.

[36] B. Sirakov, C. Tomei and A. Zaccur, Results of Ambrosetti-Prodi type for non-self-adjoint elliptic operators, Annales de l'Institut Henri Poincaré, AN 35(2018), 1757-1772.

[37] M.W. Smiley, A finite element method for computing the bifurcation function for semilinear elliptic BVPs, J. Comp. Appl. Math., 70 (1996) 311327.

[38] M. Taylor, Remarks on Fractional Diffusion Equations, www.unc.edu/ math/Faculty/met/fdif.pdf .

[39] C. Tomei and A. Zaccur, Geometric aspects of Ambrosetti-Prodi operators with Lipschitz nonlinearities, Analysis and topology in nonlinear differential equations, PNLDE 85, Springer, Cham, (2014) 445-456.

[40] B. Z. Vulikh, Cone, Encyclopedia of Mathematics, Springer.

[41] E. Wofsey, Sequential compactness of unit ball in $\mathcal{B}(X)$, available at https://math.stackexchange.com/questions/3344475/.

[42] K. Yosida, Functional Analysis, Springer, Berlin (1974).

[43] E. Zeidler, Nonlinear Functional Analysis and its Applications, IV: Applications to Mathematical Physic, Springer (1988), New York.

Marta Calanchi, Dipartimento di Matematica, Università degli Studi di Milano, Via Saldini, 5020147 Milano, Italy. marta.calanchi@unimi.it

Carlos Tomei, Departamento de Matemática, PUC-Rio R. Mq. S. Vicente 225, Rio de Janeiro 22451-900, Brazil. carlos.tomei@gmail.com 